\title{
Effect of A $\beta$ Oligomers on Neuronal APP Triggers a Vicious Cycle Leading to the Propagation of Synaptic Plasticity Alterations to Healthy Neurons
}

\author{
Marta Rolland, Rebecca Powell, Muriel Jacquier-Sarlin, ${ }^{-}$Sylvie Boisseau, Robin Reynaud-Dulaurier, \\ Jose Martinez-Hernandez, Louise André, ${ }^{-}$Eve Borel, ${ }^{(0)}$ Alain Buisson, and Fabien Lanté \\ Université Grenoble Alpes, Inserm, U1216, Grenoble Institut Neurosciences, GIN, 38000 Grenoble, France
}

\begin{abstract}
Alterations of excitatory synaptic function are the strongest correlate to the pathologic disturbance of cognitive ability observed in the early stages of Alzheimer's disease (AD). This pathologic feature is driven by amyloid- $\beta$ oligomers (A $\beta$ os) and propagates from neuron to neuron. Here, we investigated the mechanism by which Aßos affect the function of synapses and how these alterations propagate to surrounding healthy neurons. We used complementary techniques ranging from electrophysiological recordings and molecular biology to confocal microscopy in primary cortical cultures, and from acute hippocampal and cortical slices from male wild-type and amyloid precursor protein (APP) knock-out (KO) mice to assess the effects of A Bos on glutamatergic transmission, synaptic plasticity, and dendritic spine structure. We showed that extracellular application of A $\beta$ os reduced glutamatergic synaptic transmission and long-term potentiation. These alterations were not observed in APP KO neurons, suggesting that APP expression is required. We demonstrated that ABos/APP interaction increases the amyloidogenic processing of APP leading to intracellular accumulation of newly produced A $\beta$ os. Intracellular A $\beta$ os participate in synaptic dysfunctions as shown by pharmacological inhibition of APP processing or by intraneuronal infusion of an antibody raised against Aßos. Furthermore, we provide evidence that following APP processing, extracellular release of ABos mediates the propagation of the synaptic pathology characterized by a decreased spine density of neighboring healthy neurons in an APP-dependent manner. Together, our data unveil a complementary role for $\mathrm{A} \beta$ os in $\mathrm{AD}$, while intracellular $\mathrm{A} \beta$ os alter synaptic function, extracellular $\mathrm{A} \beta$ os promote a vicious cycle that propagates synaptic pathology from diseased to healthy neurons.
\end{abstract}

Key words: $\beta$ - and $\gamma$-secretase inhibition; Alzheimer's disease; APP KO mice; APP processing; NMDA-dependent synaptic transmission; synaptic plasticity

\section{Significance Statement}

Here we provide the proof that a vicious cycle between extracellular and intracellular pools of $A \beta$ oligomers (A $\beta$ os) is required for the spreading of Alzheimer's disease (AD) pathology. We showed that extracellular $A \beta$ os propagate excitatory synaptic alterations by promoting amyloid precursor protein (APP) processing. Our results also suggest that subsequent to APP cleavage two pools of $A \beta$ os are produced. One pool accumulates inside the cytosol, inducing the loss of synaptic plasticity potential. The other pool is released into the extracellular space and contributes to the propagation of the pathology from diseased to healthy neurons. Pharmacological strategies targeting the proteolytic cleavage of APP disrupt the relationship between extracellular and intracellular $\mathrm{A} \beta$, providing a therapeutic approach for the disease.

Received Oct. 21, 2019; revised Mar. 4, 2020; accepted Apr. 3, 2020.

Author contributions: M.J.-S., A.B., and F.L. designed research; M.R., R.P., M.J.-S., S.B., R.R.-D., J.M.-H., L.

A., E.B., and F.L. performed research; F.L. contributed unpublished reagents/analytic tools; M.R., R.P., M.J.-S.,

S.B., R.R.-D., L.A., A.B., and F.L. analyzed data; M.J.-S., A.B., and F.L. wrote the paper.

This work was supported by Grenoble Alpes University; Institut National de la Santé et de la Recherche Médicale; Région Auvergne-Rhône-Alpes; Grant ANR-15-IDEX-02 NeuroCoG from Agence Nationale de la Recherche (F.L.), in the framework of the "Investissements d'avenir" program and Grant ANR MALAAD program; GREnoble Excellence in Neurodegeneration (GREEN); R.P. was a fellow of Fondation pour la Recherche Médicale; and Fondation Vaincre Alzheimer. We would like to acknowledge Prof. Remy Sadoul for providing plasmids to quantify gamma secretase activity. We also thank Dr. Mireille Albrieux and Prof. Michel Vignes for critical reading of the manuscript.

J. Martinez-Hernandez's present addresses: University of the Basque Country (UPV/EHU), 48940 Leioa, Spain.

\section{Introduction}

Alzheimer's disease $(\mathrm{AD})$ is a chronic neurodegenerative disorder characterized by a progressive cognitive impairment and by the presence of the following two characteristic lesions of the cerebral cortex: neurofibrillary tangles of tau and extracellular

The authors declare no competing financial interests.

Correspondence should be addressed to Fabien Lanté at fabien.lante@univ-grenoble-alpes.fr or Alain Buisson at alain.buisson@univ-grenoble-alpes.fr.

https://doi.org/10.1523/JNEUROSCI.2501-19.2020

Copyright $\odot 2020$ the authors 
deposits of $\beta$-amyloid ( $\mathrm{A} \beta$ ) peptides as senile plaques (Cipriani et al., 2011). Extracellular senile plaques are formed from $A \beta$ peptides of different lengths, as follows: the 40-residue peptide $\mathrm{A} \beta_{40}$ represents the most abundant physiological $\mathrm{A} \beta$ isoform in the brain, while the pathogenic 42-residue $\mathrm{A} \beta_{42}$ is elevated in AD brains (Näslund et al., 1994). A $\beta$ results from the proteolytic processing of the amyloid precursor protein (APP), a transmembrane protein expressed in neuronal and non-neuronal cells in the CNS. In neurons, APPs are distributed in different subcellular compartments and processed by two routes designated as the nonamyloidogenic and amyloidogenic pathways, one precluding and the other promoting the generation of $\mathrm{A} \beta$ peptides (Haass et al., 2012). In the amyloidogenic pathway, APPs are internalized into endocytic compartments and subsequently cleaved by two proteases, $\beta$-secretase and $\gamma$-secretase, to generate $\mathrm{A} \beta$ (Kamenetz et al., 2003). APP primarily matures through the secretory pathway starting from the endoplasmic reticulum to the Golgi apparatus, where it is post-translationally modified before vesicular transport to the cell surface, though it can also be processed in the endoplasmic reticulum/intermediate compartment. $\mathrm{A} \beta$ produced in the endoplasmic reticulum is almost exclusively $\mathrm{A} \beta_{42}$ and is not destined for secretion (Greenfield et al., 1999), suggesting that several pools of $\mathrm{A} \beta$ are produced by neurons: one deposited extracellularly and the other accumulated intracellularly (LaFerla et al., 2007). Increased production of $A \beta$ is thought to be the initial causative factor leading to the alteration of cognitive function, yet little is known about the contribution of these different pools of $\mathrm{A} \beta$ to the progression of the disease.

The ability of neurons to modulate excitatory synaptic strength is believed to be a cellular correlate of learning and memory. By studying the impact of $\mathrm{A} \beta$ oligomers $(\mathrm{A} \beta \mathrm{os})$ on fast excitatory synaptic transmission mediated by the activation of postsynaptic ionotropic glutamate receptors AMPA and NMDA, we may improve our understanding of the causative effect of $A \beta$ os on cognitive processes. Extracellular application of diffusible $\mathrm{A} \beta$ os on rat hippocampal slices blocks NMDA receptor-dependent long-term potentiation (LTP; Walsh et al., 2002). Other studies have confirmed the contribution of extracellular $\mathrm{A} \beta$ os to alterations of learning and memory processes and synaptic failure (Shankar et al., 2007; Li et al., 2009; Wei et al., 2010). In contrast, several studies revealed that memory impairments in various transgenic models of $\mathrm{AD}$ are concomitant with intracellular accumulation of $\mathrm{A} \beta$ os that precedes plaque formation (Oddo et al., 2003; Bayer and Wirths, 2010). This intracellular accumulation of $\mathrm{A} \beta$ os is also observable in brains of $\mathrm{AD}$ patients with learning deficits (Gouras et al., 2000), suggesting that this specific feature is causal in the alteration of memory processes.

The present study aims at further understanding the impact of these two pools of $\mathrm{A} \beta$ os on the excitatory neurotransmission. We reveal that extracellular $\mathrm{A} \beta$ os promote APP processing and a subsequent accumulation of intracellular $\mathrm{A} \beta$ os that is responsible for the synaptic dysfunctions.

\section{Materials and Methods}

\section{Animals}

All experiments were conducted in accordance with the European Community Council directives of November 24, 1986 (86/609/EEC) and with the French guidelines on the use of living animals in scientific investigations. Experiments were performed with male Swiss mice from Janvier, male wild-type (WT) C57BL/6 and male Amyloid Precursor Protein Knock-Out (KO) C57BL/6 mice (APP ${ }^{\mathrm{tm} 1 \mathrm{Dbo}}$; The Jackson Laboratory).

\section{Preparation of human amyloid- $\beta$ solution}

The peptide used in these experiments was obtained from human recombinant $\mathrm{A} \beta_{1-42}$ peptide (Bachem) resuspended in 1,1,1,3,3,3-hexafluoro-2-propanol (HFIP) to $1 \mathrm{~mm}$ until complete resuspension (Stine et al., 2003). $\mathrm{A} \beta$ os were prepared by diluting $\mathrm{A} \beta_{1-42}$ peptide to $1 \mathrm{~mm}$ in DMSO then to $100 \mu \mathrm{M}$ in ice-cold HEPES and bicarbonate-buffered saline solution or in artificial CSF (ACSF; in mм: $\mathrm{NaCl} \mathrm{119,} \mathrm{KCl} 2.5$, $\mathrm{NaH}_{2} \mathrm{PO}_{4} 1.25, \mathrm{MgSO}_{4} 1.3, \mathrm{CaCl}_{2} 2.5, \mathrm{NaHCO}_{3} 26$, and glucose 11) with immediate vortexing and bath sonication and then incubated at $4^{\circ} \mathrm{C}$ for $24 \mathrm{~h}$ with mild agitation. Final solutions of $\mathrm{A} \beta$ os were prepared by diluting the solution at $100 \mu \mathrm{M}$ in ACSF, DMEM, or intracellular solution.

\section{Purification of $\mathrm{A} \beta$ monomer}

The A $\beta$ monomer is purified on a C18 column ( $200 \mu \mathrm{l}, 5 \mathrm{mg}$; SPEChromabond-HRX C18 ec, Macherey-Nagel). The column was equilibrated with $0.1 \%$ trifluoroacetic acid (TFA) in water. Immediately after dilution in DMSO, the $\mathrm{A} \beta$ sample was loaded and the column was washed three times with $0.1 \%$ TFA. Then, a gradient of acetonitrile from $30 \%$ to $60 \%$ was applied. Fractions $(0.1 \mathrm{ml})$ were collected. The elution profile was determined by measuring the absorbance at $275 \mathrm{~nm}$. The peak fraction was collected, and the concentration of peptide was determined by absorbance at $275 \mathrm{~nm}$ using $\epsilon 275 \mathrm{~nm}=1400 \mathrm{M}^{-1} \mathrm{~cm}^{-1}$. The peptide is then stored at $-80^{\circ} \mathrm{C}$.

\section{Production of histidine-tagged proteins}

To make the plasmids for the fusion protein [A $\beta$-His (histidine)] of murine amyloid protein and the secreted soluble form of APP (sAPP-His), the cDNA containing the sequence for murine $\mathrm{A} \beta_{1-42}$ and human sAPP $695 \alpha$ were obtained from synthetic oligonucleotides (containing a NdeI restriction site as forward primers and a PspXI restriction site as reverse primers; Sigma-Aldrich) using overlapping PCR. PCR products were then cloned into a pet28a-vector (Novagen, Merck-Millipore) and subsequently constructed as HIS-murine- $\mathrm{A} \beta$-expressing plasmid (pet28a-murine-A $\beta_{1-42}$ ) and HIS-sAPP $\alpha$-expressing plasmid (pet28asAPP $\alpha$ ). The resulting plasmids were verified by sequencing Escherichia coli BL21 (DE3) was transformed with the fusion protein plasmids (for either murine-A $\beta_{1-42}$ or $\operatorname{sAPP} \alpha$ ) and a single colony chosen to grow a $250 \mathrm{ml}$ starter culture in Luria broth (LB medium) overnight at $37^{\circ} \mathrm{C}$. The next day, the $10 \mathrm{ml}$ of culture was diluted in $1 \mathrm{~L}$ of LB culture medium. When the culture reached an $\mathrm{OD}_{600}$ of 0.8 , isopropyl- $\beta$-D-thiogalactopyranoside was added to $1 \mathrm{~mm}$ concentration for induction. The culture was grown for an additional $4 \mathrm{~h}$, and the cells harvested by centrifugation at $4000 \times g$ for $20 \mathrm{~min}$. The cell was resuspended in $10 \mathrm{ml}$ of ice-cold PBS and lysed by sonication at ice-cold temperature. The cell extract was then centrifuged at $20,000 \times g$ for $15 \mathrm{~min}$ at $4^{\circ} \mathrm{C}$. For $\operatorname{sAPP} \alpha$ purification, the supernatant was kept, whereas it was discarded for murine- $\mathrm{A} \beta_{1-42}$. In this case, the pellet was resuspended in $10 \mathrm{ml}$ of $8 \mathrm{M}$ urea in PBS and sonicated as previously described before centrifugation at $20,000 \times g$ for $15 \mathrm{~min}$ at $4^{\circ} \mathrm{C}$. The supernatant $(5 \mathrm{ml})$ was diluted with $15 \mathrm{ml}$ of binding buffer (PBS with $10 \mathrm{~mm}$ imidazole at $\mathrm{pH}$ 8.0). Before affinity purification using nickel-nitriloacetic acid (NTA) column purification, samples were filtered on $0.45 \mu \mathrm{m}$. The Ni-NTA column $(3 \mathrm{ml}$ of Protino Ni-NTA Agarose; Macherey-Nagel) was equilibrated with binding buffer before loading the sample on the column. Then the column was washed with the washing buffer (PBS with $30 \mathrm{~mm}$ imidazole at $\mathrm{pH}$ 8.0) with 5-10 column volumes. The protein was then eluted with the elution buffer (PBS with $500 \mathrm{~mm}$ imidazole at $\mathrm{pH}$ 7.4). The absorbance at $280 \mathrm{~nm}$ was used to monitor the elution, but the concentration of the fusion proteins was estimated by comparing the intensity of the band of the protein on SDS-PAGE with that of a known quantity of BSA. A final concentration of $100 \mu \mathrm{M}$ was obtained, and aliquots were stored at $-80^{\circ}$ C. Aliquots from all subsequent purification steps were analyzed by SDS-PAGE, and the identities of sAPP $\alpha$ and murine $\mathrm{A} \beta_{1-42}$ were verified by Western blot using monoclonal antibodies against the $\mathrm{N}$-terminal domain of APP (22C11) or A $\beta$ sequence (4G8), respectively.

\section{Cell lines}

Mouse neuroblastoma N2a were cultured in DMEM (Sigma-Aldrich) supplemented with $10 \%$ fetal bovine serum (Millipore Sigma), as previously described (Gouras et al., 2010). 


\section{Primary culture of cortical neurons}

Primary cortical neurons were prepared from Swiss embryonic mice [embryonic day 14 (E14) to E16), as previously described (Léveillé et al., 2008). Cerebral cortices were dissected, dissociated, and cultured in DMEM containing $5 \%$ fetal bovine serum, $5 \%$ horse serum, and $2 \mathrm{~mm}$ glutamine (all from Millipore Sigma) on 24-well plates (Falcon Becton Dickinson Labware Europe) for biochemical experiments. Neurons were seeded on $12 \mathrm{~mm}$ coverslips (Dominique Dutscher). Dishes and coverslips were coated with $0.1 \mathrm{mg} / \mathrm{ml}$ poly-D-lysine and $0.02 \mathrm{mg} / \mathrm{ml} \mathrm{laminin}$ (Sigma-Aldrich). Cultures were maintained at $37^{\circ} \mathrm{C}$ in a humidified atmosphere containing 5\% $\mathrm{CO}_{2}-95 \%$ air (Frandemiche et al., 2014) for $13-15 \mathrm{~d}$ in vitro (DIV) before use.

\section{Brain slices preparation}

Brain slices were prepared from 20- to 30-d-old mice for patch-clamp recordings and from 3-month-old mice for extracellular recordings. The brains of wild-type Swiss, wild-type C57BL/6 and APP KO mice were removed quickly, and $300-\mu \mathrm{m}$-thick sagittal slices containing both cortex and hippocampus were cut in the following ice-cold cutting solution (in mM): $\mathrm{KCl} 2.5, \mathrm{NaH}_{2} \mathrm{PO}_{4} 1.25, \mathrm{MgSO}_{4} 10, \mathrm{CaCl}_{2} 0.5, \mathrm{NaHCO} 326$, sucrose 234 , and glucose 11 , saturated with $95 \% \mathrm{O}_{2}$ and $5 \% \mathrm{CO}_{2}$ ) with a Leica VT1200 blade microtome (Leica Microsystems). After the dissection, slices were kept in oxygenated ACSF at $37 \pm 1^{\circ} \mathrm{C}$ for $30 \mathrm{~min}$ and then kept at room temperature for at least $1 \mathrm{~h}$ before recordings.

\section{Electrophysiological recordings}

For patch-clamp experiments, cortical neurons from cultures or somatosensory layer 5 pyramidal neurons were visualized in a chamber on an upright microscope with transmitted illumination and continuously perfused at $2 \mathrm{ml} / \mathrm{min}$ with an oxygenated $\mathrm{Mg}^{2+}$-free ACSF as follows (in mM): $\mathrm{NaCl} 119, \mathrm{KCl} 2.5, \mathrm{NaH}_{2} \mathrm{PO}_{4} 1.25, \mathrm{CaCl}_{2} 2.5, \mathrm{NaHCO}_{3} 26$, and glucose 11, at room temperature. Spontaneous EPSCs (sEPSC) were recorded at a membrane potential of $-60 \mathrm{mV}$ with borosilicate glass pipettes of 4-5 M $\Omega$ resistance filled with $\sim 30 \mu \mathrm{l}$ of an intracellular solution, as follows (in mM): $117.5 \mathrm{CsMeSO}_{4}, 15.5 \mathrm{CsCl}, 10 \mathrm{TEACl}, 8 \mathrm{NaCl}, 10$ HEPES, 0.25 EGTA, $4 \mathrm{MgATP}$, and 0.3 NaGTP, at pH 7.3. Signals were acquired using a double EPC 10 Amplifier (HEKA Elektronik) filtered at $2 \mathrm{kHz}$, sampled at $10 \mathrm{kHz}$, and analyzed with Patchmaster software (HEKA Elektronik). Recordings were considered stable when the input and access resistances did not change $>20 \%$ during the experiment. To isolate either NMDA or AMPA/kainite sEPSCs, we used $\mathrm{Mg}^{2+}$-free ACSF containing 2,3-dihydroxy-6-nitro-7-sulfonyl-benzo[f]quinoxaline (NBQX; $10 \mu \mathrm{M}$ ), a potent inhibitor of non-NMDA glutamate receptor channels exhibiting IC $_{50}$ values of $0.1-0.4 \mu \mathrm{M}$ for AMPA and 1.7-8 $\mu \mathrm{M}$ for kainate receptors (Traynelis et al., 2010) that does not present any cross-reactivity for NMDA receptors (Goldstein and Litwin, 1993) or 2amino-5-phosphonovalerate (D-APV; $100 \mu \mathrm{M}$ ) an NMDA receptor antagonist with an $\mathrm{IC}_{50}$ values of $0.28 \mu \mathrm{M}$ (NR2A), $0.46 \mu \mathrm{M}$ (NR2B), and $1.6 \mu \mathrm{M}$ (NR2C), respectively. In control condition, we recorded sEPSCs in $\mathrm{Mg}^{2+}$-free ACSF $5 \mathrm{~min}$ after whole-cell configuration had been achieved (referred as $\mathrm{T}_{0}$ ) and $20 \mathrm{~min}$ after $\mathrm{T}_{0}$ (referred to as $\mathrm{T}_{20}$ ). In the extracellular $\mathrm{A} \beta$ os $(\mathrm{eA} \beta \mathrm{os})$ condition, we recorded sEPSCs in $\mathrm{Mg}^{2+}$ free ACSF at $\mathrm{T}_{0}$ and $\mathrm{T}_{20}$, a perfusion with $\mathrm{Mg}^{2+}$-free ACSF containing $\mathrm{A} \beta$ os $(300 \mathrm{nM})$. Protocol was similar for D-APT $(5 \mu \mathrm{M})$ and $\beta$-secretase inhibitor $(1 \mu \mathrm{M})$. In the intracellular $\mathrm{A} \beta$ os (iA $\beta$ os) condition, the intracellular solution contained $300 \mathrm{~nm} A \beta$ os. In this condition, we recorded sEPSCs in $\mathrm{Mg}^{2+}$-free ACSF at $\mathrm{T}_{0}$ and $\mathrm{T}_{20}$. The protocol was similar for 4G8 antibody diluted into the whole-cell recording intracellular solution (1:100 dilution). The final concentration of $4 \mathrm{G} 8$ antibody inside the patch pipette was $10 \mu \mathrm{g} / \mathrm{ml}$. sEPSC and miniature EPSCs (mEPSC) analyses were performed on recordings of $180 \mathrm{~s}$ at $-60 \mathrm{mV}$. The amplitude threshold was set at $8 \mathrm{pA}$, and all the detected events were accepted or rejected on the basis of visual examination. The average frequencies and amplitudes of these events were expressed in picoamperes for sEPSC amplitudes and in hertz for sEPSC frequencies and as percentage of the ratio between values measured at $\mathrm{T}_{20}$ over values at $\mathrm{T}_{0}$.

For LTP experiments, hippocampus was extracted from the slice and transferred in the microscope chamber. Oxygenated ASCF (in mM: $\mathrm{NaCl}$ 119, $\mathrm{KCl} 2.5, \mathrm{NaH}_{2} \mathrm{PO}_{4} 1.25, \mathrm{MgSO}_{4} 1.3, \mathrm{CaCl}_{2} 2.5, \mathrm{NaHCO}_{3} 26$, and glucose 11) was continuously perfused into the chamber $(2 \mathrm{ml} / \mathrm{min})$ at $28^{\circ} \mathrm{C}$. A borosilicate glass pipette filled with ACSF was attached to the measuring electrode, and a stimulation electrode was also mounted. To induce field EPSP (fEPSP) in the hippocampal CA1 region, the stimulating electrode was placed on the Schaffer collaterals, and the recording electrode was positioned in the striatum radiatum. Test stimuli were delivered once every $15 \mathrm{~s}$, and the stimulus intensity was adjusted to produce $40-50 \%$ of the maximal response. A stable baseline was recorded for at least $15 \mathrm{~min}$. LTP was induced by theta burst stimulation (involving five trains with 10 bursts of four pulses delivered at $100 \mathrm{~Hz}$, an interburst interval of $200 \mathrm{~ms}$, and a $20 \mathrm{~s}$ interval between each train). Signals were amplified with a double EPC 10 Amplifier (HEKA Elektronik). $\mathrm{eA} \beta$ os and $\beta$-secretase inhibitor were added to the perfusion ACSF 20 min before theta burst stimulation. Data in millivolts are normalized and expressed as a percentage of baseline.

\section{Immunoblotting for neuronal lysates}

At 13-15 DIV, cortical neurons in culture were treated with $\mathrm{A} \beta \mathrm{os}$ and $\beta$-secretase inhibitor intravenously (at $1 \mu \mathrm{M}$; Calbiochem) for $30 \mathrm{~min}$. After treatment, neurons were rapidly transferred on ice and rinsed twice with ice-cold PBS then washed with PBS containing 0.1\% saponin and rinsed again with ice-cold PBS. Neurons were then lysed with RIPA buffer (50 mu Tris-HCl, pH 7.4; $150 \mathrm{~mm} \mathrm{NaCl} ; 1 \%$ Triton X-100; $1 \%$ sodium deoxycholate; $0.1 \%$ SDS; $1 \mathrm{~mm}$ EDTA) containing a cocktail of protease and phosphatase inhibitors $1 \%(\mathrm{v} / \mathrm{v})$. Lysates were dosed for proteins using the BCA method. Samples in loading buffer were boiled for $5 \mathrm{~min}$, and equal amounts of protein $(20 \mu \mathrm{g})$ were resolved on $4-20 \%$ gradient Bis-Tris polyacrylamide precast gels (Bio-Rad) in denaturing conditions. Proteins were transferred to a polyvinylidene difluoride $0.2 \mu \mathrm{m}$ membranes (Millipore) for $2 \mathrm{~h}$ at $4^{\circ} \mathrm{C}$. Membranes were blocked with Tris-buffered saline (10 mm Tris and $150 \mathrm{~mm} \mathrm{NaCl}, \mathrm{pH}$ 7.4) containing $0.01 \%$ Tween 20 and 5\% nonfat dry milk for $1 \mathrm{~h}$ at room temperature. Membranes were then incubated overnight at $4^{\circ} \mathrm{C}$ with the following primary antibodies: APP full-length (1:2000 dilution; catalog \#22C11, Millipore); APP C-terminal fragments (CTFs; 1:2000 dilution; catalog \#Y188, Abcam); actin (1:2000 dilution; catalog \#A2066, SigmaAldrich); and A $\beta$ peptide (1:1000; catalog \#4G8, Covance). Membranes were incubated with the appropriate horseradish peroxidase-conjugated secondary antibodies (1:40,000; Jackson ImmunoResearch and Immunotech) for $45 \mathrm{~min}$ at room temperature. Specific proteins were visualized with an enhanced chemiluminescence ECL Detection System (Bio-Rad). Chemiluminescence detection was performed with the Bio-Rad Chemidoc system and analyzed with the ImageJ software. In these experiments, $A \beta$ os were used at $100 \mathrm{~nm}$.

\section{Membrane/cytosol fractionation}

To analyze subcellular distribution of APP fragments, we performed a membrane versus soluble/cytosolic fractionation protocol adapted from Florean et al. (2008). Briefly, cells were permeabilized for 3 min by $50 \mu \mathrm{M}$ digitonin in an intracellular saline solution (in $\mathrm{mm}: 130 \mathrm{~mm} \mathrm{KCl}, 10 \mathrm{~mm}$ $\mathrm{NaCl}, 20 \mathrm{~mm}$ HEPES, $1 \mathrm{~mm} \mathrm{MgSO}_{4}$, and $5 \mathrm{~mm}$ succinate, at $\mathrm{pH}$ 7.2) containing $50 \mu \mathrm{M}$ EGTA and protease/phosphatase cocktail inhibitors (Roche/Sigma). The supernatant was then collected and centrifuged for $30 \mathrm{~min}$ at $100,000 \times g$ at $4^{\circ} \mathrm{C}$. The resulting supernatant was kept as a soluble/cytosolic fraction. The neurons in the wells (membrane fractions) were washed twice with PBS before being collected in RIPA buffer.

\section{Plasmids}

pFR-Luciferase (pFR-Luc) reporter vector containing the Firefly Luciferase (FR-Luc) gene under the control of the yeast GAL4 activation sequence, phRL-thymidine kinase (TK) vector containing the renilla luciferase gene (phRL-TK) and pRC-CMV vector containing a cDNA encoding for human APP695 fused to the yeast transcription factor GAL4 (APP695-Gal4) were provided by Prof. R. Sadoul (Grenoble Institut des Neurosciences) and were previously described by Hoey et al. (2009).

The strategy used to obtain the double fluorescently tagged human APP695 Swedish (hAPPswe) mutant chimera was adapted from Sannerud et al. (2011). Using the mcherry-APP-HA-EYFP (enhanced yellow fluorescent protein) pcDNA3 vector (from the laboratory of Wim Annaert, Center for Brain and Disease Research, Leuven, Belgium), 
which contains the fluorescent mCherry within the ectodomain sequence of human APP695, we performed site-directed mutagenesis to introduce the following mutations (NL833/834 to KM) just before the A $\beta$ sequence according to the manufacturer instructions (Phusion SiteDirected Mutagenesis Kit, Thermo Fisher Scientific). The resulting mcherry-hAPPswe-HA-EYFP pcDNA3 vector was verified by sequencing.

cDNAs of WT human APP695 (APPwt) and the Swedish mutant (APPswe) were cloned into pmcherry-N1 vector (SnapGene) using the BamHI and AgeI restriction sites. All constructions in pmCherry vector were verified by sequencing.

\section{Neuronal transfection}

Transfections were performed on cortical neuron cultures after 12 DIV with Mirus TransIT-2020 Transfection Reagent (Euromedex) according to the instructions of the manufacturer. Briefly, for each condition in a 24-well plate, $1 \mu \mathrm{g}$ of plasmid containing mcherry-hAPPswe-HA-EYFP pcDNA3 vector was mixed with $1.5 \mu$ of transit-2020 reagent in $50 \mu \mathrm{l}$ of serum-free growth medium and incubated for $30 \mathrm{~min}$ at room temperature. Then the mixture was applied to cells, and cultures were returned to the incubator for $48 \mathrm{~h}$.

Two-step transfection was used to study the impact of $\mathrm{A} \beta$ overproduction in the neighboring healthy cells. Three micrograms of WT human APP695 (APPwt-mCherry) or the Swedish mutant (APPswemCherry) plasmids [mixed with $1 \mathrm{M} \mathrm{CaCl} 2$ and HEPES-buffered saline (HBS) buffer] were first applied to cells for $30 \mathrm{~min}$. Then, $3 \mu \mathrm{g}$ of LifeActin-GFP plasmid (mixed with $1 \mathrm{M} \mathrm{CaCl} 2$ and HBS buffer) was added to the cells for $40 \mathrm{~min}$. Transfection medium was replaced with conditioned growth medium, and cultures were returned to the incubator until use at DIV 14-15.

Dual-Glo luciferase reporter gene activity assay for quantification of $\gamma$-secretase activity

$\gamma$-Secretase activity was measured with the Dual-Glo luciferase reporter gene (Gralle et al., 2009). Twenty-four hours after seeding the clone of $\mathrm{N} 2 \mathrm{a}$ into $3.5 \mathrm{~cm}$ dishes, cells were cotransfected with pFR-Luc $(30 \mathrm{ng})$, APP695-Gal4 (300 ng), and phRL-TK (5 ng) using the cationic polymer transfection reagent Exgen (Euromedex) according to the protocol of the manufacturer. Dual-Glo luciferase activity assays were performed 48 $\mathrm{h}$ after transfection by the quantification of firefly and Renilla luciferase activities (Promega). In all experiments, Firefly Luciferase activity was normalized using the constitutive Renilla luciferase activity. In these experiments $\mathrm{A} \beta$ os were used at $100 \mathrm{~nm}$.

\section{Immunostaining}

After $48 \mathrm{~h}$ of transfection, cortical neurons were cultured for $2 \mathrm{~h}$ in serum-free growth medium and treated or not with $A \beta$ os $100 \mathrm{~nm}$ for $30 \mathrm{~min}$. Cultured were then fixed with $4 \%$ paraformaldehyde $/ 4 \%$ sucrose in $0.1 \mathrm{M} \mathrm{PBS}$, at $\mathrm{pH} 7.3$ for $10 \mathrm{~min}$ at $37^{\circ} \mathrm{C}$. Cells were washed in PBS and processed for immunostaining. After saturation and permeabilization in $3 \%$ BSA $/ 0.1 \%$ Triton X-100 in 0.1 m PBS for 30 min, cells were incubated with primary antibodies for $2 \mathrm{~h}$, washed three times in $0.1 \%$ Triton $\mathrm{X}$ 100 in $0.1 \mathrm{M}$ PBS, and incubated with Life Technologies Alexa Fluor 647conjugated secondary antibody (1:1000; Thermo Fisher Scientific) for 1 h. After three washes in $0.1 \%$ Triton X-100 in 0.1 m PBS, cells were incubated for 5 min with Hoechst stain (catalog \#33258, Sigma-Aldrich) for nuclei staining and mounted with DAKO fluorescent mounting medium (DAKO). To characterize subcellular compartments, the following specific antibodies were used: mouse monoclonal anti-EEA1 (1:500; G-4, Santa Cruz Biotechnology) for early endosomes, mouse monoclonal anti-LAMP2 antibody (1:500; Proteintech) for lysosomes and mouse monoclonal anti-58K Golgi protein (1:500; 58K-9, GeneTex) for Golgi apparatus.

\section{Confocal imaging of transfected neurons}

Transfected neurons were washed twice with HBSS solution and then fixed with $4 \%$ paraformaldehyde $/ 5 \%$ sucrose in $0.1 \mathrm{M}$ PBS, at $\mathrm{pH} 7.3$ for $10 \mathrm{~min}$ at room temperature. Cells were washed in PBS and mounted in fluorescent mounting medium. Preparations were examined on a Zeiss LSM 710 confocal laser-scanning microscope, and images were acquired with a Zeiss Airyscan module with an oil-immersion Plan Apochromat
A

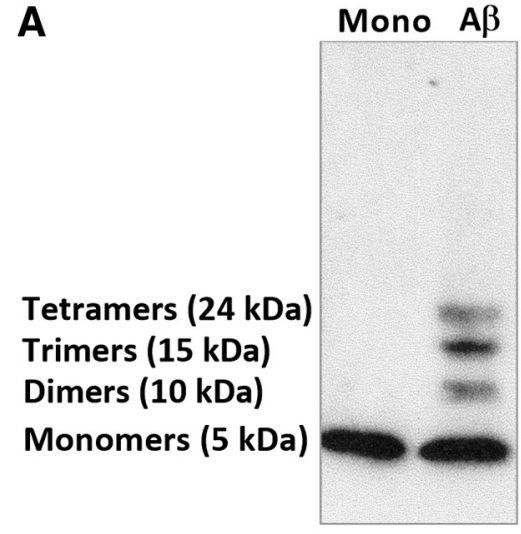

B

Acetonitrile gradient (\%)

S FT $1020 \quad 304050$

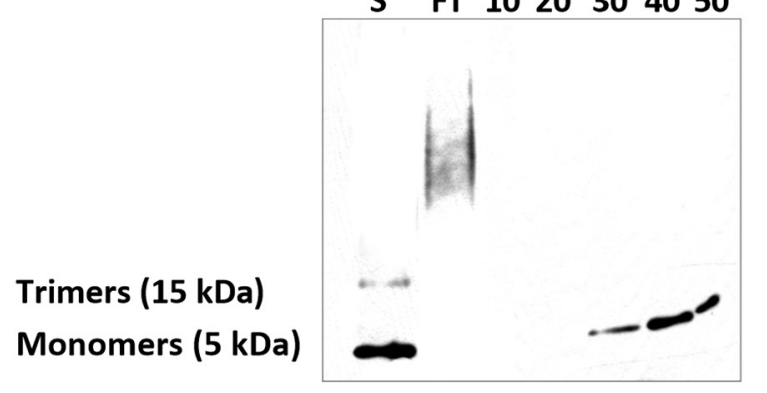

C

NI I FT W E1 E2 E3 E4

\section{Tetramers $(24 \mathrm{kDa})$ Trimers (15 kDa)}

Monomers (5 kDa)

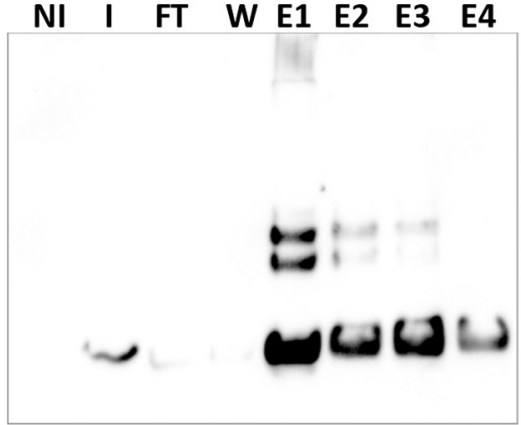

Figure 1. Analysis of experimental $A \beta$ oligomers solution. $A$, Western blot analysis of experimental solutions of $A \beta$ that display a composition of monomers and various oligomeric forms of $A \beta$ (dimers to tetramers). $B$, SDS-PAGE analysis of $A \beta$ monomers after purification on the C18 column. All fractions were electrophoresed on $15 \%$ tris-glycine gel. A $\beta$ monomers are mainly eluted at $30 \%$ acetonitrile. S, Sample loaded; FT, flow through, peptides eluted at $30 \%, 40 \%$, and $50 \%$ acetonitrile. C, SDS-PAGE analysis of murine A $\beta$ after purification on affinity chromatography on a Nickel column. All fractions were electrophoresed on a $15 \%$ tris-glycine gel. $\mathrm{A} \beta$ monomers are eluted in PBS containing $250 \mathrm{~mm}$ imidazole. Solutions of murine $A \beta$ display a composition of monomers, trimers, and tetramers. $\mathrm{Nl}$, Noninduced bacterial extract; I, induced bacterial extract FT, flow through; W, washes, peptides eluted in the four first fractions.

$63 \times$ objective (numerical aperture 1.46) to improve lateral resolution $(\sim 140 \mathrm{~nm})$ and signal-to-noise ratios. For illustration, images were merged using ImageJ software (RRID:SCR_003070). Spine density was assessed by using NeuronStudio software, which performed an automatic tracing and reconstruction of neuron structures from confocal image stacks (Icahn School of Medicine at Mount Sinai, New York, NY).

\section{Experimental design and statistical analysis}

Statistical analyses were performed with GraphPad version 6.0 software. After determining whether the data follow normal distribution or not with a Shapiro-Wilk normality test, we performed, for normally distributed data, one-way or two-way ANOVA followed by Tukey's multiplecomparisons test. If the data did not display a normal distribution, we 
A
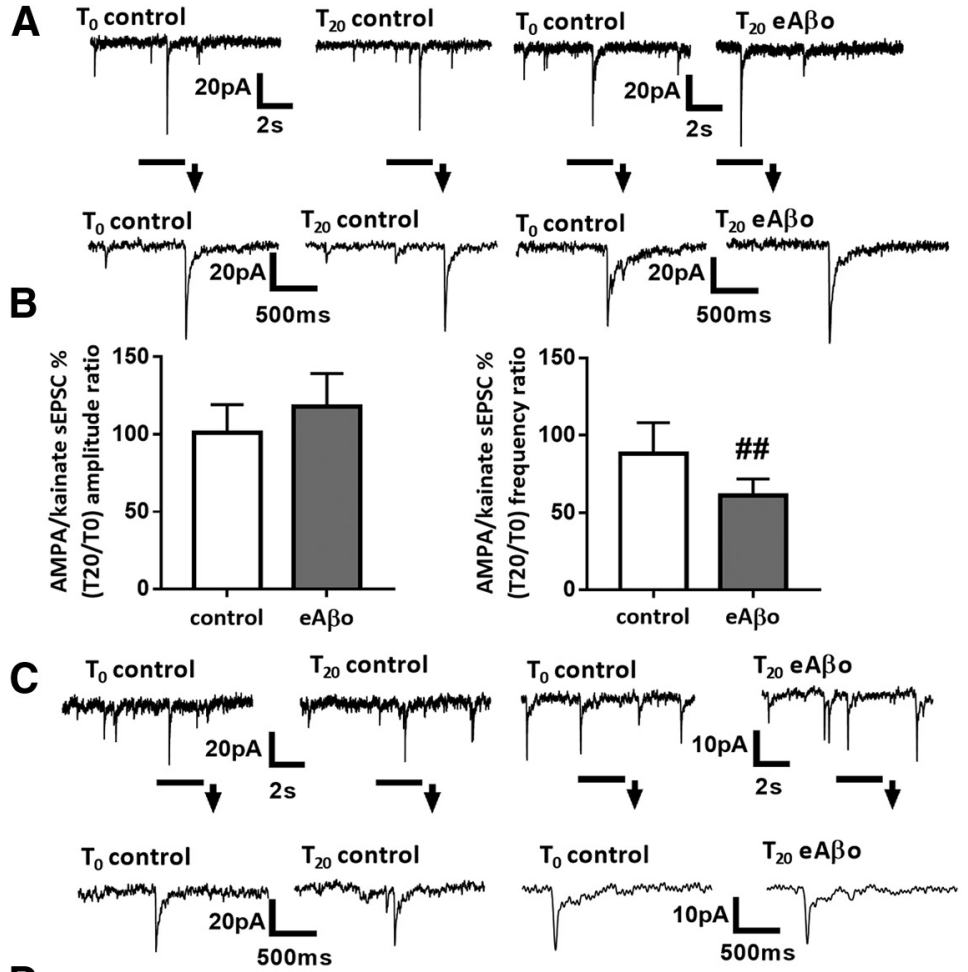

D
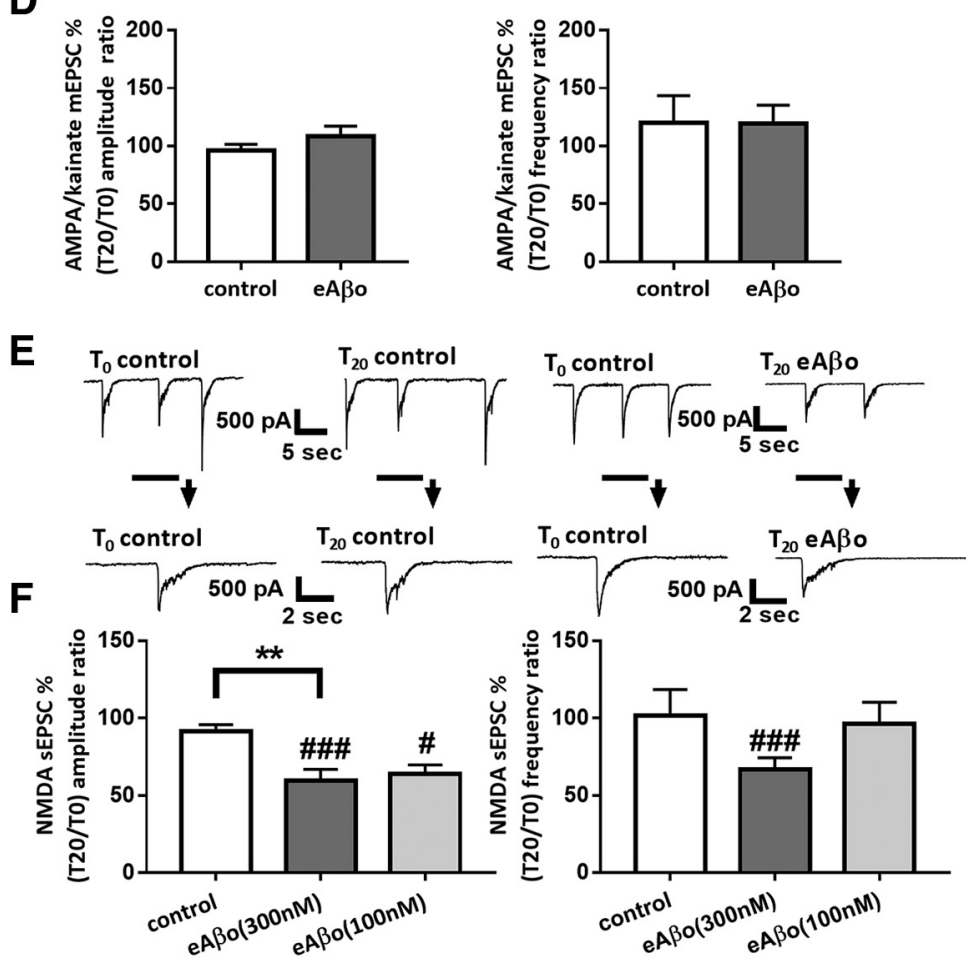

Figure 2. eA $\beta$ os perturb spontaneous synaptic activity in cultures of mouse cortical neurons. $A$, Representative traces of AMPA/kainate sEPSCS at $\mathrm{T}_{0}$ and $\mathrm{T}_{20}$ in control condition or with eA $\beta 0 \mathrm{OS}(300 \mathrm{nM}$ ). $\boldsymbol{B}$, Bar graphs (mean \pm SEM) showing the $T_{20} / T_{0}$ ratio of AMPA/kainate $S E P S C$ amplitudes and frequencies in control condition (white bars; $n=10$ neurons, Wilcoxon $W=-17(19,-36), p=0.4316$, for sEPSC amplitudes; and Wilcoxon $W=-2(13,-15) p=0.9375$ for sEPSC frequencies); with eA $\beta$ os (gray bars; $n=10$ neurons, Wilcoxon $W=-15(20,-35), p=0.4922$, for sEPSC amplitudes; and Wilcoxon $W=-49(3,-52), p=0.0098$, for sEPSC frequencies). Control versus eA $\beta 0$ s (Mann-Whitney $U=44(99,111), p=0.6842$, for sEPSC amplitudes; and Mann-Whitney $U=38(117,93), p=0.3923$, for sEPSC frequencies). C, Representative traces of AMPA/kainate mEPSCs at $\mathrm{T}_{0}$ and $\mathrm{T}_{20}$ in control condition or with eA $\beta$ os $(300 \mathrm{~nm})$. $\boldsymbol{D}$, Bar graphs (mean \pm SEM) showing the $\mathrm{T}_{20} / \mathrm{T}_{0}$ ratio of AMPA/kainate $\mathrm{mEPSC}$ amplitude and frequency in control condition (white bars; $n=7$ neurons, Wilcoxon $W=-14(7,-21), p=0.2969$, for mEPSC amplitudes; and Wilcoxon $W=4$ $(5,-1), p=0.5000$, for mEPSC frequencies) or with eA $\beta$ os (gray bars; $n=6$ neurons, Wilcoxon $W=15(30,-15)$, $p=0.42$, for mEPSC amplitudes; and Wilcoxon $\mathrm{W}=10(19,-9), p=0.46$, for mEPSC frequencies). Control versus performed a Kruskal-Wallis followed by Dunn's multiple-comparisons test as the nonparametric test. Paired comparisons were assessed by Wilcoxon signed-rank tests and Mann-Whitney test for unpaired comparisons. Results are expressed as the mean \pm SEM from independent experiments performed separately and corresponding to different cell cultures. Significance was set at 0.05 . For electrophysiology experiments " $n$ " corresponds to the number of cells recorded, and " $N$ " to the number of mice used.

Drugs

NBQX, D-APV; (2R)-amino-5-phosphonopentanoate); $N$-[N-(3,5-difluorophenacetyl)-L-alanyl]-(S)-phenylglycine t-butyl ester (DAPT), $\beta$-secretasepi inhibitor IV, and tetrodotoxin (TTX) were from Sigma-Aldrich. 4G8 antibody from BioLegend was diluted (1:100) into the recording solution and infused $20 \mathrm{~min}$ before the recording. The final concentration of 4G8 inside the pipette was $10 \mu \mathrm{g} / \mathrm{ml}$.

\section{Results}

Extracellular A $\beta$ os perturb spontaneous synaptic activity in primary cultures of mouse cortical neurons

We studied the effect of eA $\beta$ os at $300 \mathrm{~nm}$ on glutamatergic neurotransmission in murine primary cortical neurons. A $\beta$ oligomers were prepared from commercially available peptides and resuspended in artificial CSF. When we performed a Western blot of this solution, we observed the presence of lowmolecular weight oligomers of $\mathrm{A} \beta$ from dimers to tetramers (Fig. 1). As shown in Figure 2, $A$ and $B$, eA $\beta$ os did not modify AMPA/kainate sEPSC amplitudes $\left(\mathrm{T}_{0}=\right.$ $23.35 \pm 5.93 \mathrm{pA} ; \mathrm{T}_{20}=23.55 \pm 4.62 \mathrm{pA} ; \mathrm{T}_{20}$ vs $\mathrm{T}_{0}: 117.68 \pm 21.5 \% ; p=0,492 ; n=10$ neurons) but significantly decreased AMPA/

eA $\beta$ os (Mann-Whitney $U=19(47,106), p=0.13$, for mEPSC amplitudes; and Mann-Whitney $U=34(64,89), p=0.96$, for mEPS( frequencies). $\boldsymbol{E}$, Representative traces of NMDA sEPS(s at $\mathrm{T}_{0}$ and $\mathrm{T}_{20}$ in control condition or with eA $\beta$ os (300 nM). $\boldsymbol{F}$, Bar graphs (mean \pm SEM) showing the $T_{20} / T_{0}$ ratio of NMDA sEPSC amplitudes and frequencies in control condition (white bars; $n=13$ neurons, Wilcoxon $W=-55(18,-73), p=0.0574$, for sEPSC amplitudes; and Wilcoxon $W=-21(12,-33), p=$ 0.2383 for sEPSC frequencies); with eA $\beta$ os ( $300 \mathrm{~nm}$; gray bars; $n=17$ neurons, Wilcoxon $W=-137(8,-145), p=0.0004$, for sEPSC amplitudes; and Wilcoxon $W=-97(4,-101)$, $p=0.0009$, for sEPSC frequencies) or with eA $\beta 0$ s (100 nm; light gray bars; $n=7$ neurons, Wilcoxon $W=-28(0,-28)$, $p=0.0156$, for sEPSC amplitudes; and Wilcoxon $W=-1(1$, $-2), p>0.9999$, for sEPSC frequencies). One-way ANOVA and Tukey's post hoc test for multiple comparisons $\left(F_{(2,34)}=6.937\right.$; $p=0.0030$; control vs eA $\beta 0$ s ( $300 \mathrm{~nm}), p=0.0028)$ for NMDA $s$ EPSC amplitudes and Kruskal-Wallis followed by Dunn's multiple-comparisons test $(6.473 ; p=0.0393)$ for NMDA sEPSC frequencies. ${ }^{* *} p<0.01$; \#p $<0.05$, \#\#p $<0.01$, \#\# $<0.001$ relative to the $\mathrm{T}_{0}$ recording normalized to $100 \%$. 
kainate sEPSC frequencies $\left(\mathrm{T}_{0}=0.59 \pm\right.$ $0.10 \mathrm{~Hz} ; \mathrm{T}_{20}=0.37 \pm 0.10 \mathrm{~Hz} ; \mathrm{T}_{20}$ vs $\mathrm{T}_{0}$ : $60.71 \pm 10.9 \% ; p=0.009 ; n=10$ neurons). Next, we evaluated the effect of eA $\beta$ os on spontaneously occurring miniature excitatory postsynaptic current (mEPSC) recorded in the presence of TTX that blocks action potential formation and propagation. In contrast to sEPSCs, we did not detect any change on AMPA/kainate mEPSC frequencies $\left(\mathrm{T}_{0}=0.35 \pm 0.09 \mathrm{~Hz}\right.$; $\mathrm{T}_{20}=0.37 \pm 0.08 \mathrm{~Hz} ; \mathrm{T}_{20}$ vs $\mathrm{T}_{0}: 92.32 \pm$ $6.5 \% ; p=0.3750 ; n=10$ neurons; Fig. $2 C$, $D)$. This result suggested that the reduced frequencies of sEPSCs recorded under eA $\beta$ os is due to a reduction of neuronal excitability and not to a modification of presynaptic glutamate release probability. Interestingly, the application of eA $\beta$ os markedly lowered both the amplitudes $\left(\mathrm{T}_{0}=432.94 \pm 63.98 \mathrm{pA} ; \mathrm{T}_{20}=252.38 \pm\right.$ $51.62 \mathrm{pA} ; \mathrm{T}_{20}$ vs $\mathrm{T}_{0}: 59.08 \pm 7.4 \% ; p=$ $0.0004 ; n=17)$ and the frequencies $\left(\mathrm{T}_{0}=\right.$ $0.15 \pm 0.03 \mathrm{~Hz} ; \mathrm{T}_{20}=0.07 \pm 0.02 \mathrm{~Hz} ; \mathrm{T}_{20}$ vs $\mathrm{T}_{0}: 66.65 \pm 7.6 \% ; p=0.0009 ; n=17$; Fig. $2 E$, $F)$ of NMDA sEPSCs. In contrast, a lower concentration of eA $\beta$ os (100 nM) was still able to reduce NMDA sEPSC amplitudes $\left(\mathrm{T}_{20}\right.$ vs $\left.\mathrm{T}_{0}: 63.74 \pm 5.98 \% ; p=0.015 ; n=7\right)$ but failed to decrease NMDA sEPSC frequencies $\left(\mathrm{T}_{20}\right.$ vs $\mathrm{T}_{0}: 96.03 \pm 14.17 \% ; p>$ 0.99 ; $n=7$; Fig. $2 F$ ), suggesting a dose-dependent effect of eA $\beta$ os on sEPSC frequencies. These data indicated that a $20 \mathrm{~min}$ exposure of eA $\beta$ os altered neuronal excitability and selectively reduced NMDA sEPSC amplitudes in primary culture of cortical neurons. Perturbations of cellular excitability have already been reported in transgenic models of $\mathrm{AD}$ or after $\mathrm{A} \beta \mathrm{os}$ application (Brown et al., 2011; Marcantoni et al., 2014; Tamagnini et al., 2015). Our next objective was to investigate how eA $\beta$ os selectively perturb NMDA sEPSC amplitudes.

\section{eAßos reduce NMDA sEPSC amplitudes in cortical slice neurons from WT but not APP KO mice}

Previous studies highlighted the role of APP in extracellular A $\beta$ os-mediated toxicity and dysfunction (Lorenzo et al., 2000; Shaked et al., 2006, 2009; Fogel et al., 2014; Puzzo et al., 2017; Wang et al., 2017). In this regard, we investigated whether APP is required for eA $\beta$ os altering NMDA-dependent synaptic transmission by using cortical slices from WT and APP KO mice. eA $\beta$ os markedly reduced the amplitudes $\left(\mathrm{T}_{0}=43.75 \pm 7.83 \mathrm{pA} ; \mathrm{T}_{20}=29.48 \pm 5.05 \mathrm{pA} ; \mathrm{T}_{20}\right.$ vs $\mathrm{T}_{0}$ : $72.71 \pm 6 \% ; p=0.0017 ; n=16$ neurons; $N=8$ mice) and the frequencies $\left(\mathrm{T}_{0}=0.82 \pm 0.13 \mathrm{~Hz} ; \mathrm{T}_{20}=0.53 \pm 0.07 \mathrm{~Hz} ; \mathrm{T}_{20}\right.$ vs $\mathrm{T}_{0}$ : $71.88 \pm 9.7 \% ; p=0.029 ; n=16$ neurons; $N=8$ mice; Fig. $3 A, B)$ of

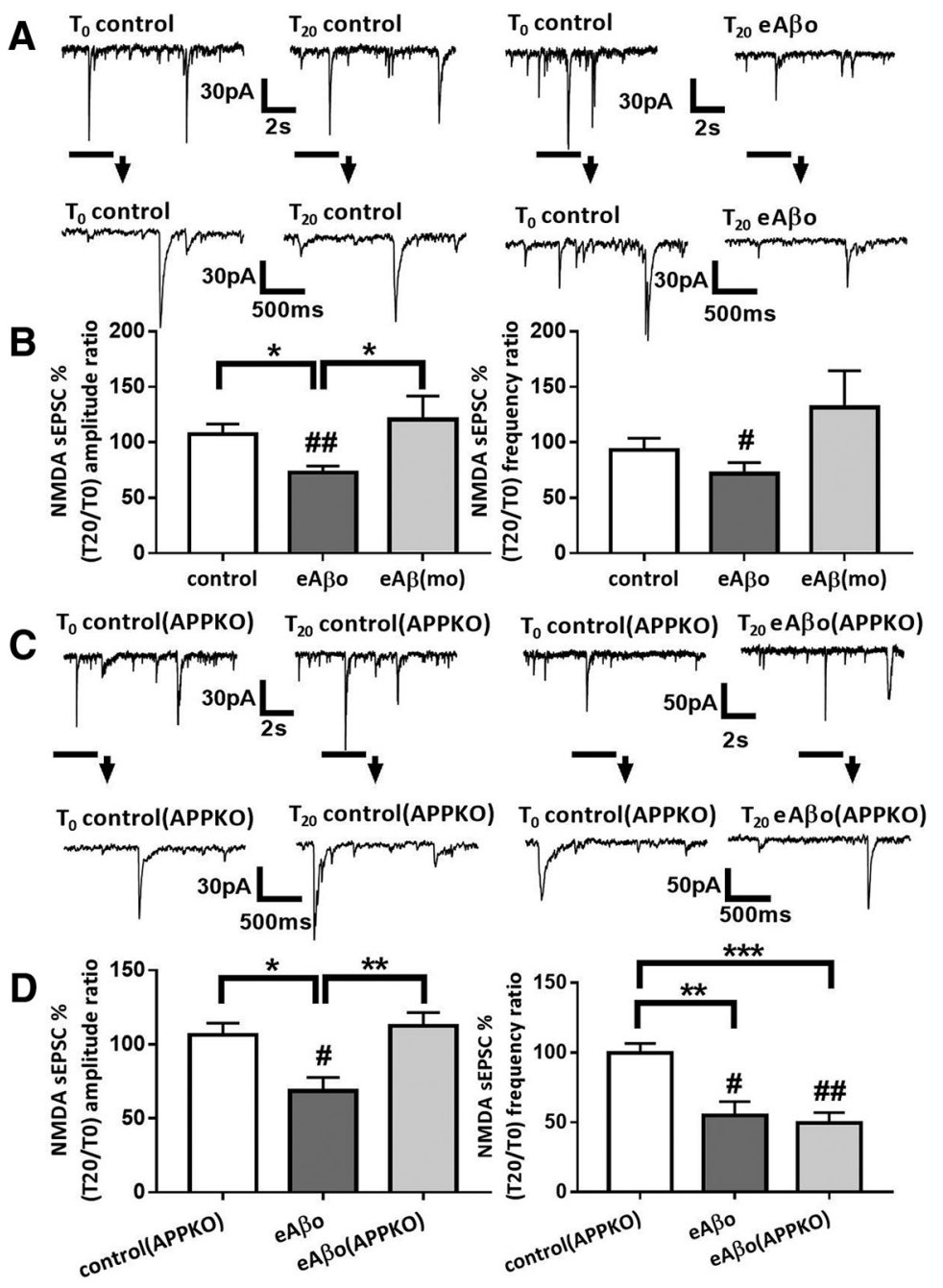

Figure 3. eA $\beta$ os reduce NMDA sEPSC amplitude in cortical slice neurons from WT but not from APP KO mice. $A$, Representative traces of NMDA sEPSCS recorded in WT neurons from Swiss mice at $T_{0}$ and $T_{20}$ in control condition; with eA $\beta$ OS. $B$, Bar graphs (mean \pm SEM) showing the $T_{20} / T_{0}$ ratio of NMDA sEPSC amplitude and frequency recorded in WT neurons from Swiss mice in control condition (white bars; $n=11$ neurons, $N=9$ mice, Wilcoxon $W=22(44,-22)$, $p=0.3652$, for sEPSC amplitudes; and Wilcoxon $W=-16(25,-41), p=0.5195$, for sEPSC frequencies); with eA $\beta 0$ s (gray bars; $n=16$ neurons, $N=8$ mice, Wilcoxon W $=-114(11,-125), p=0.0017$, for sEPSC amplitudes; and Wilcoxon $W=-84(26,-110), p=0.0290$ for sEPSC frequencies); with eA $\beta$ monomers [eA $\beta(\mathrm{mo}), 300 \mathrm{~nm}$; light gray bars; $n=8$ neurons, $N=4$ mice, Wilcoxon $W=6(21,-15), p=0.7422$, for sEPSC amplitudes; and Wilcoxon $W=8(22,-14)$, $p=0.6406$, for $s$ EPSC frequencies). One-way ANOVA and Tukey's post hoc test for multiple comparisons $\left(F_{(2,32)}=5.578\right.$, $p=0.0084$; control vs eA $\beta 0$ s; $p=0.0423$, eA $\beta$ os vs eA $\beta(\mathrm{mo}), p=0.0143$ ] for NMDA sEPSC amplitudes and Kruskal Wallis followed by Dunn's multiple-comparisons test $(5.076, p=0.0790$, for NMDA sEPSC frequencies. $C$, Representative traces of NMDA sEPSCs recorded in APP KO neurons at $T_{0}$ and $T_{20}$ in control condition, with eA $\beta 0$ s. D. Bar graphs (mean \pm SEM) showing the $T_{20} / T_{0}$ ratio of NMDA sEPSC amplitude and frequency recorded in APP KO neurons in control condition (white bars; $n=6$ neurons, $N=6$ mice, Wilcoxon $W=1(11,-10), p>0.9999$, for sEPSC amplitudes; and Wilcoxon $W=-3(9,-12), p=0.8438$, for sEPSC frequencies); in WT neurons from C57BL/6J mice with eA $\beta 0$ (gray bars; $n=6$ neurons, $N=3$ mice, Wilcoxon $W=-21(0,-21), p=0.0313$, for sEPSC amplitudes; and Wilcoxon $W=-21(0,-21)$, $p=0.0313$, for sEPSC frequencies); in APP K0 neurons with eA $\beta$ os (light gray bars; $n=8$ neurons, $N=5$ mice, Wilcoxon $W=18(27,-9), p=0.2500$, for sEPSC amplitudes; and Wilcoxon $W=-36(0,-36), p=0.0078$, for sEPSC frequencies). One-way ANOVA and Tukey's post hoc test for multiple comparisons $\left[F_{(2,17)}=6.792 ; p=0.0068\right.$; control(APP K0) vs eA $\beta$ os $p=0.0298$; eA $\beta$ os vs eA $\beta$ os (APP K0), $p=0.0072$ ] for NMDA sEPSC amplitudes, and one-way ANOVA followed by Tukey's post hoc test for multiple comparisons $\left[F_{(2,18)}=11.64 ; p=0.0006\right.$; control(APP KO) vs eA $\beta 0$ s, $p=0.0041$; control(APP K0) vs eA $\beta$ os (APP K0), $p=0.0008$ ] for NMDA sEPSC frequencies. ${ }^{*} p<0.05$, ${ }^{* *} p<0.01$, ${ }^{* *} p<0.001$; $\# p<0.05$, \#\#p $<0.01$ relative to the $\mathrm{T}_{0}$ recording normalized to $100 \%$.

NMDA sEPSCs recorded in neurons from Swiss WT mice, confirming the data obtained in primary cultured neurons. Moreover, the lack of effect of extracellular application of $\mathrm{A} \beta$ monomers $(300 \mathrm{nM})$ on both the amplitudes $\left(\mathrm{T}_{0}=34.43 \pm\right.$ $4.87 \mathrm{pA} ; \mathrm{T}_{20}=37.71 \pm 5.48 \mathrm{pA} ; \mathrm{T}_{20}$ vs $\mathrm{T}_{0}: 120.71 \pm 19.92 \%$; 
$p=0.74 ; n=8$ neurons; $N=4$ mice $)$ and the frequencies $\left(\mathrm{T}_{0}=\right.$ $0.67 \pm 0.17 \mathrm{~Hz} ; \mathrm{T}_{20}=0.72 \pm 0.21 \mathrm{~Hz} ; \mathrm{T}_{20}$ vs $\mathrm{T}_{0}: 131.18 \pm 31.39 \%$; $p=0.64 ; n=8$ neurons; $N=4$ mice; Fig. $3 B$ ) of NMDA sEPSCs suggested that the alterations are specially associated with eA $\beta$ os. In contrast to $\mathrm{C} 57 \mathrm{BL} / 6 \mathrm{~J}$ wild-type mice, where eA $\beta$ os reduced both the amplitudes $\left(\mathrm{T}_{0}=28.62 \pm 5.19 \mathrm{pA} ; \mathrm{T}_{20}=\right.$ $19.67 \pm 5.19 \mathrm{pA} ; \mathrm{T}_{20}$ vs $\mathrm{T}_{0}: 68.44 \pm 9.19 \% ; p=0.031 ; n=6$ neurons; $N=3$ mice) and the frequencies $\left(\mathrm{T}_{0}=0.71 \pm\right.$ $0.26 \mathrm{~Hz} ; \mathrm{T}_{20}=0.35 \pm 0.17 \mathrm{~Hz} ; \mathrm{T}_{20}$ vs $\mathrm{T}_{0:} 54.76 \pm 9.95 \%$; $p=0.031 ; n=6$ neurons; $N=3$ mice; Fig. $3 D$ ) of NMDA sEPSCs, we did not detect any perturbations in the amplitudes of currents recorded in neurons from APP KO mice $\left(\mathrm{T}_{0}=\right.$ $30.72 \pm 6.88 \mathrm{pA} ; \mathrm{T}_{20}=34.22 \mathrm{pA} \pm 8.07 \mathrm{pA} ; \mathrm{T}_{20}$ vs $\mathrm{T}_{0}: 112.35$ $\pm 9.07 \% ; p=0.25 ; n=8$ neurons, $N=5$ mice), while we still observed a decrease in the frequencies $\left(\mathrm{T}_{0}=0.57 \pm 0.20 \mathrm{~Hz}\right.$; $\mathrm{T}_{20}=0.19 \pm 0.06 \mathrm{~Hz} ; \mathrm{T}_{20}$ vs $\mathrm{T}_{0}: 49.34 \pm 7.64 \% ; p=0.007 ;$ $n=8$ neurons; $N=5$ mice; Fig. $3 C, D)$. Thus, the reduction of NMDA sEPSC amplitudes by eA $\beta$ os required the presence of APP. The reduction of NMDA sEPSC frequencies appeared to be independent of APP expression.

\section{Neurons overproducing secreted toxic $A \beta$ affect neighboring} neurons through APP

Several studies have shown that $\mathrm{A} \beta$ overproduction in one cell leads to $\mathrm{A} \beta$ secretion into the extracellular space affecting neighboring healthy cells and could explain the spreading of the pathology across the brain (Wei et al., 2010). Here, we examined the effects of a WT human APP695 (APPwt-mcherry) or the Swedish mutant (APPswe-mCherry), overexpressing neuron, which leads to increased secreted $\mathrm{A} \beta$ in the extracellular space (data not shown) on a healthy nearby neuron. The rationale behind this experiment is to see whether the amount of secreted A $\beta$ correlates with the defects induced on the healthy neighboring neuron. For this purpose, we first transfected APPwtmCherry or APPswe-mCherry plasmids in cultured cortical neurons then, $30 \mathrm{~min}$ later, we added LifeActin-GFP (LA-GFP), a peptide that specifically binds filamentous actin. This allowed us to have one neuron expressing both LA-GFP and one of the APP-mCherry (APP neuron) next to a neuron that only expresses LA-GFP (healthy neuron; Fig. 4A). We then examined the spine density of the dendrites of the healthy neuron depending on their distance from the APP neuron. Results showed that neurons overproducing secreted $\mathrm{A} \beta$, namely APPwt-mCherry and APPswe-mCherry overexpressing neurons, decrease the spine density of the nearby healthy neuron. Indeed, the APPwtmCherry neuron significantly decreased spine density of a healthy neuron from 0 (dendrites from both neurons are overlapping) to $20 \mu \mathrm{m}$ (distance from APP neuron). APPswemCherry neuron had an even stronger impact on healthy neuron by decreasing its spine density from a range of $0-40 \mu \mathrm{m}$. These results suggest that, in order for the pathology to propagate from cell to cell, A $\beta$ has to be secreted (Fig. $4 B$ ). Furthermore, the distance gradient of the synaptotoxic effect in neurons expressing APPswe suggests a relationship between the synaptotoxicity and the secretion level of $\mathrm{A} \beta$.

Furthermore, we wanted to assess the role of APP in this spreading of synaptotoxic effects. We conducted the same set of experiments using APP knock-out primary cultured cortical neurons. Here, one APP KO neuron is overexpressing both APPswe-mCherry and LA-GFP, and the neighboring healthy APP KO neuron is only overexpressing LA-GFP. In these experiments, we did not observe any spreading of synaptotoxic effects induced by the APPswe-mCherry neuron on the surrounding neurons, suggesting the implication of APP expression in the spreading of the pathology (Fig. $4 B$ ).
A
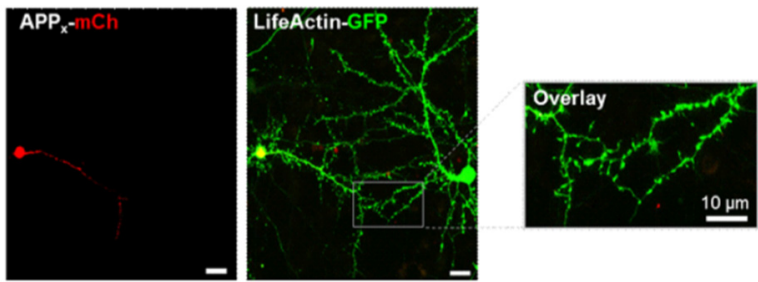

B

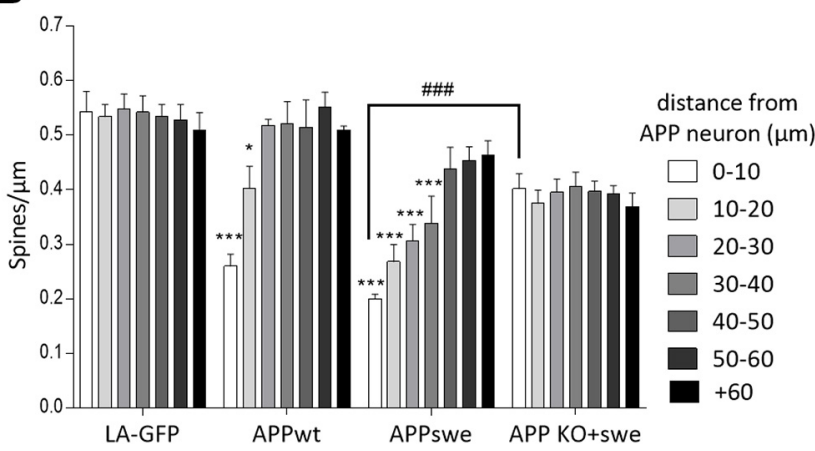

Figure 4. Overexpression of APPwt. APPswe in cortical cell cultures decreases the spine density of neighboring healthy wild-type neuron, in an APP-dependent manner. $\boldsymbol{A}$, Representative confocal images of cultured cortical neurons where the neuron on the left is overexpressing either LA-GFP only (LA-GFP), APPwt-mCh (APPwt), APPswe-mCh (APPswe), or APP KO neurons that overexpress APPswe-mCh (APP KO+swe), and the neuron on the right is only overexpressing LA-GFP (healthy neuron). Scale bar, $10 \mu \mathrm{m}$. $\boldsymbol{B}$, Bar graphs (mean \pm SEM) show the spine density of healthy neurons depending on the distance from (LAGFP, APPwt, APPswe, or APP KO+swe) neurons ( $n=$ at least 3 neurons/condition from three different cultures). Two-way ANOVA and Tukey's post hoc test for multiple comparisons. Spine density of healthy neurons according to the distance from the APP-overexpressing neuron $\left(F_{(6,168)}=5.309 ; p<0.0001\right.$; treatment: $F_{(5,168)}=51.6, p<0.0001$, interaction: $\left.F_{(30,168)}=3.484, p<0.0001\right)$. From 0 to $10 \mu \mathrm{m}$ : LA-GFP versus APPwt, $p<0.0001$; LA-GFP vs APPswe, $p<0.0001$; APPswe vs APP K0+ swe, $p<0.0001$. From 20 to $30 \mu \mathrm{m}$ : LA-GFP vs APPwt, $p=0.0487$; LA-GFP vs APPswe, $p<0.0001$. From 30 to $40 \mu \mathrm{m}$ : LA-GFP vs APPswe, $p<0.0001$. ${ }^{*} p<0.05,{ }^{* *} p<0.001$ when compared with control condition (both neurons only overexpress LA-GFP) at equivalent distance. \#\#\# $<0.001$ when healthy neurons in APP KO plus the swe condition are compared with healthy neurons in the APPswe condition.

\section{eAßos induce APP processing through the amyloidogenic pathway}

To gain more insight into the functional relationship between eA $\beta$ os and APP, we blotted endogenous full-length APP and its cleavage products from primary cultures of cortical neurons exposed to eA $\beta$ os for $30-360 \mathrm{~min}$ (Fig. $5 \mathrm{~A}$ ). A $30 \mathrm{~min}$ exposure to eA $\beta$ os was sufficient to produce proteolytic CTFs while $\mathrm{A} \beta$ peptides were only detected after $6 \mathrm{~h}$ of $\mathrm{eA} \beta$ os exposure. According to these results, we then investigated the effects of $\mathrm{eA} \beta$ os on APP processing by analyzing the production of CTFs in presence of $\beta$-secretase inhibitors (Fig. $5 B$ ). The ratio of APP proteolytic CTFs over total full-length APP increased when primary cortical neurons were exposed to eA $\beta$ os $(1.71 \pm 0.16$ a.u.) compared with the control condition $(0.78 \pm 0.037$ a.u.; $p<0.0001$; $n=7$ independent experiments). This increased proteolytic cleavage of APP due to A $\beta$ os was abolished by $\beta$-secretase inhibitor at 1 $\mu \mathrm{M}$ ( $0.63 \pm 0.015$ a.u.; $p=0.68 ; n=4$ independent experiments) compared with control (Fig. $5 C$ ). These data suggested that eA $\beta$ os exposure promotes an increase of APP processing.

To further investigate the functional relationship between eA $\beta$ os and APP, we used a cell-based gene reporter assay to monitor $\gamma$-secretase-mediated cleavage of APP (Hoey et al., 2009). This technique uses a reporter APP695 fused at its C terminal to the transcription factor Gal4 (APP695-Gal4). The 
A
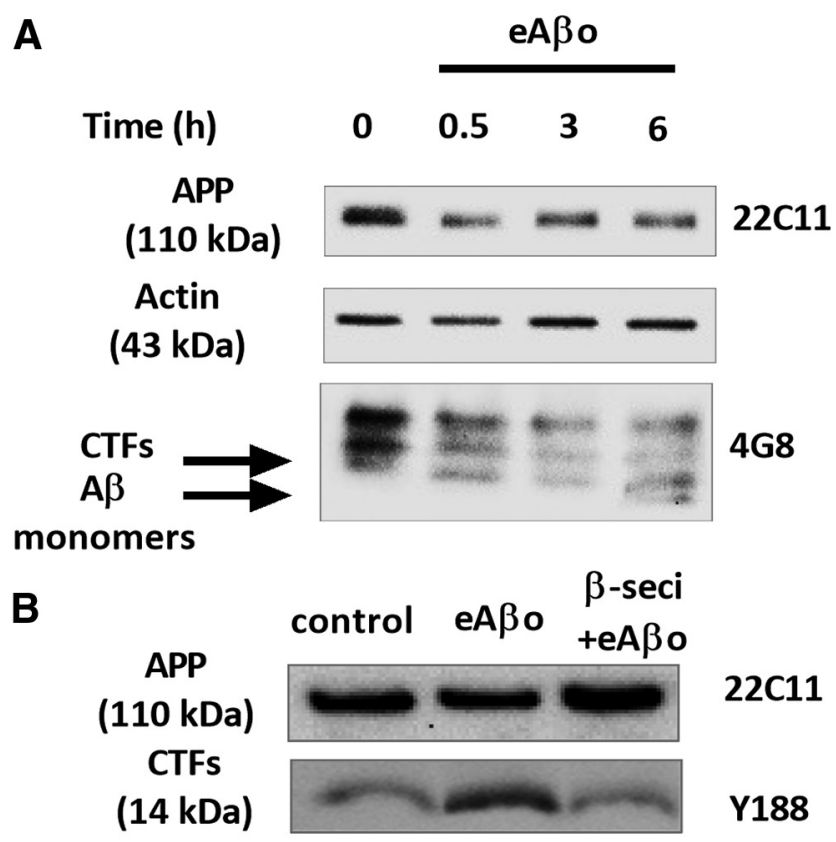

$22 C 11$ Actin
$(43 \mathrm{kDa})$

$4 G 8$

Actin (43 kDa)

$22 \mathrm{C11}$

C

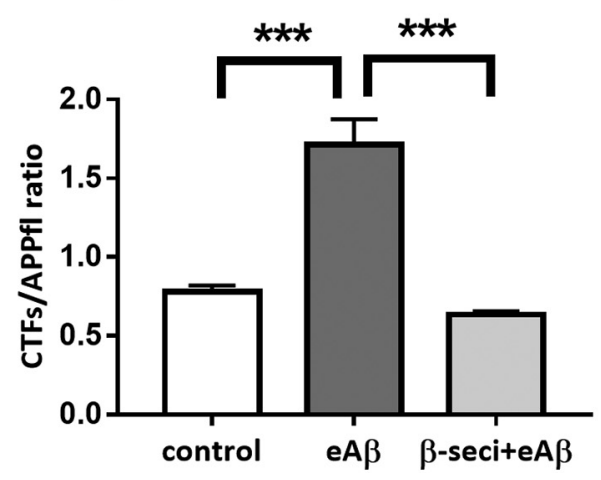

Figure 5. eA $\beta 0$ s induce APP processing through amyloidogenic pathway. A, Representative Western blot of endogenous APP and its proteolytic fragments in a whole lysate extract of cortical neurons (14 DIVs) exposed to eA $\beta$ os for $30 \mathrm{~min}$. B, Top, Representative Western blot of endogenous APP and CTFs in a whole-lysate extract of cortical neurons (14 DIVs) exposed to eA $\beta$ os with or without $\beta$-secretase inhibitor ( $\beta$-seci; $1 \mu \mathrm{m}$ ) for $30 \mathrm{~min}$. C, Quantification of APP full-length (APPfl) and APP proteolytic CTFs in control ( $n=7$ independent experiments), in the presence of eA $\beta$ os ( $n=7$ independent experiments), in the presence of eA $\beta$ os plus $\beta$-secretase inhibitor ( $n=4$ independent experiments). Results (mean \pm SEM) are expressed as the ratio of APP (TFs over full-length APP. One-way ANOVA and Tukey's post hoc test for multiple comparisons $\left(F_{(2,15)}=27.83\right.$, $p<0.0001$; control vs eA $\beta$ os, $p<0.0001$; eA $\beta$ os vs $\beta$-seci $+\mathrm{eA} \beta$ os $p<0.0001$. ${ }^{* * *} p<0.001$.

proteolytic cleavage of APP by $\alpha$ - or $\beta$ - and $\gamma$-secretases produces an amyloid precursor protein intracellular domain-Gal4 fragment that can translocate into the nucleus in which Gal4 induces transcription of a transfected Gal4-dependent Firefly Luciferase reporter gene. $\gamma$-Secretase activity was quantified by normalizing FR-Luc luminescence using the constitutive RenillaTK luciferase activity, which depends on the number of transfected cells. Preliminary experiments were performed to validate the $\gamma$-secretase test. These controls consisted in transfected cells with only FR-Luciferase and RL-TK Luciferase. In the presence of APP695-Gal4, Firefly Luciferase activity is $\sim 380$ times stronger than the control, reflecting a clear increase in $\gamma$-secretase activity $(p<0.0001 ; n=4$ independent experiments; Fig. $6 A)$. In

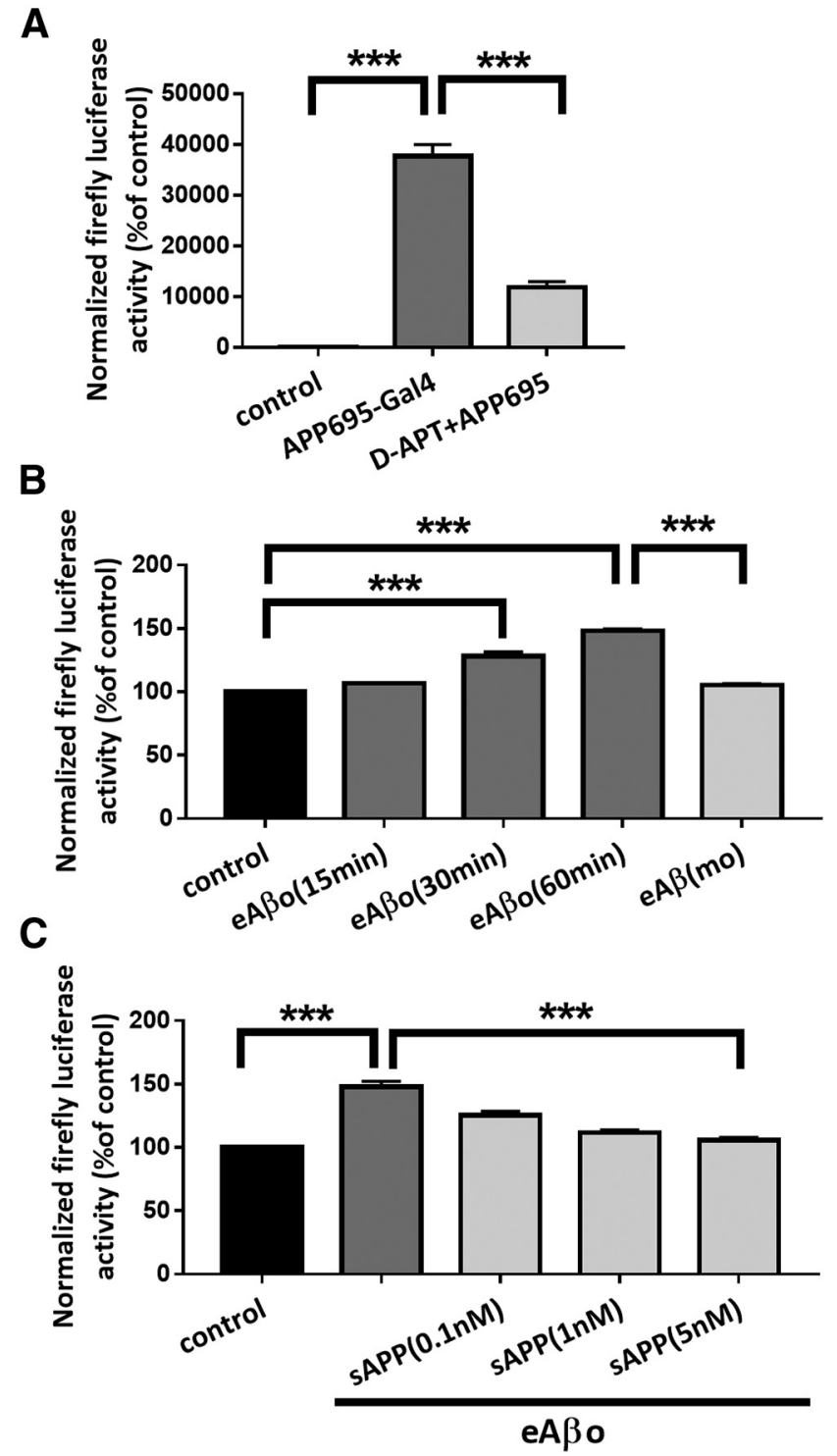

Figure 6. eA $\beta$ os promote APP processing through $\gamma$-secretase activity. $A$, Quantification of Firefly Luciferase activity when N2a cells were cotransfected with pFR-Luc Firefly Luciferase reporter gene plasmid and phRL-TK Renilla Luciferase plasmid (control; $n=4$ independent experiments); cotransfected with pFR-Luc Firefly Luciferase reporter gene plasmid, phRL-TK Renilla luciferase plasmid, and with a plasmid coding for APP695-Gal4 $(n=4$ independent experiments); cotransfected with pFR-Luc Firefly Luciferase reporter gene plasmid, phRL-TK Renilla Luciferase plasmid, and with a plasmid coding for APP695-Gal4 in the presence of DAPT ( $5 \mu \mathrm{m} ; n=4$ independent experiments). Results are expressed as a percentage of control (N2a not cotransfected with APP695-Gal4). One-way ANOVA and Tukey's post hoc test for multiple comparisons $\left(F_{(2,9)}=174.3, p<0.0001\right.$; control vs APP695-Gal4, $p<0.0001$; APP695-Gal4 vs DAPT +APP695, $p<0.0001)$. B, Quantification of Firefly Luciferase activity when N2a cotransfected with pFR-Luc Firefly Luciferase reporter gene plasmid, phRL-TK Renilla Luciferase plasmid, and a plasmid coding for APP695-Gal4 were treated with eA $\beta$ os for 15,30 , and $60 \mathrm{~min}$ ( $n=4$ independent experiments) and with eA $\beta(\mathrm{mo})$ for $60 \mathrm{~min}$ ( $n=3$ independent experiments). Results are expressed as a percentage of control (N2a not exposed to eA $\beta$ os). One-way ANOVA and Tukey's post hoc test for multiple comparisons $\left[F_{(4,14)}=139.9, p<0.0001\right.$; control vs eA $\beta$ os $(30 \mathrm{~min}), p<0.0001$; control vs eA $\beta$ os (60 min), $p<0.0001$; eA $\beta$ os ( $60 \mathrm{~min}$ ) vs eA $\beta(\mathrm{mo}), p<0.0001]$. C, Quantification of Firefly Luciferase activity when N2a cells cotransfected with pFR-Luc firefly luciferase reporter gene plasmid, phRL-TK Renilla luciferase plasmid, and a plasmid coding for APP695Gal4 were pretreated with various concentration of secreted soluble APP fragment (SAPP) and then exposed with eA $\beta$ os for $60 \mathrm{~min}$ ( $n=3$ independent experiments). Results are expressed as a percentage of control (N2a not exposed to eA $\beta$ os). One-way ANOVA and Tukey's post hoc test for multiple comparisons $\left[F_{(4,10)}=138.9, p<0.0001\right.$; control vs eA $\beta 0$ s, $p<0.0001$; eA $\beta$ os vs SAPP(5 nM), $p<0.0001]$. ${ }^{* *} p<0.001$. 

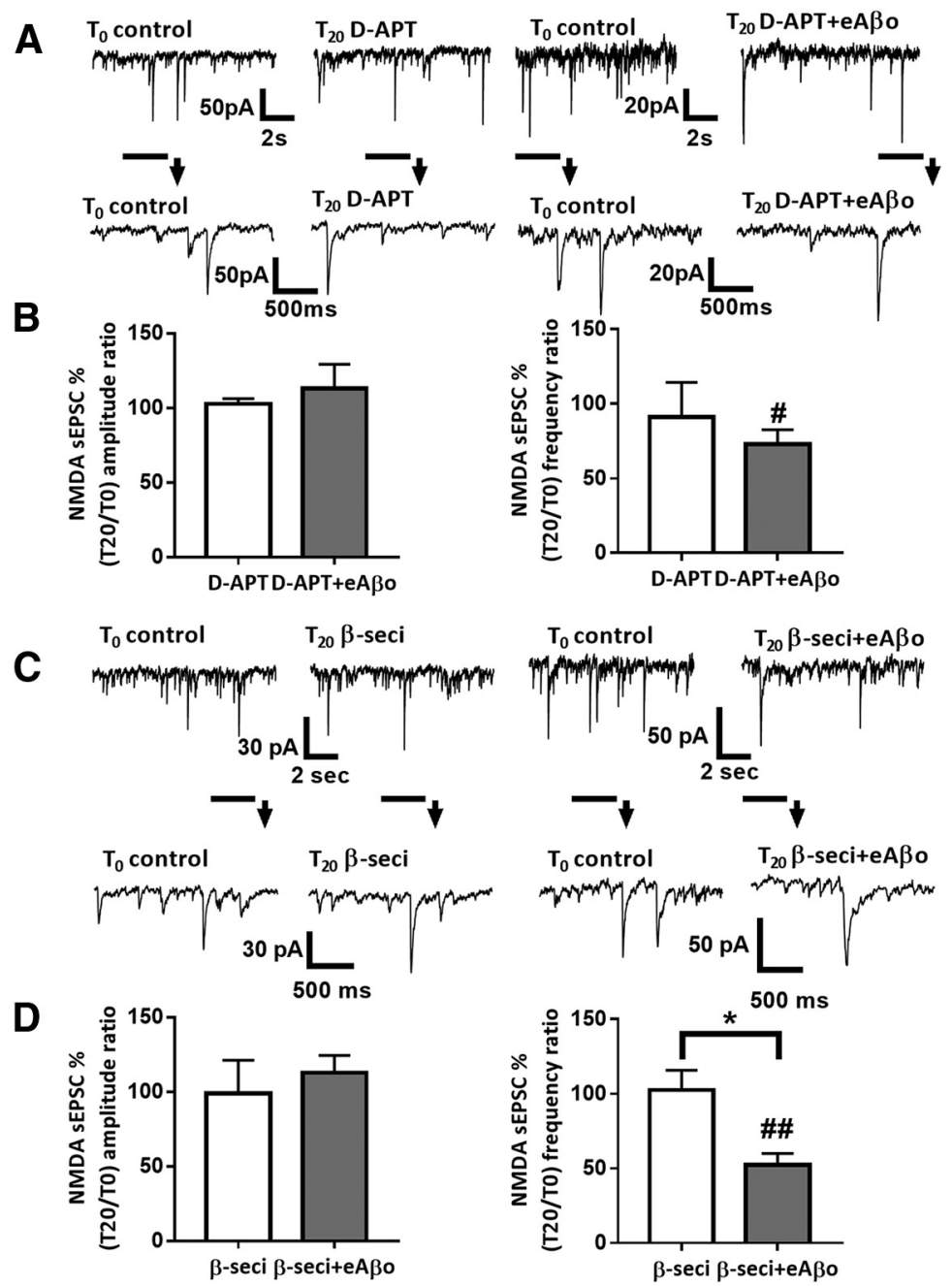

Figure 7. Alteration of NMDA sEPSCs amplitude in cortical slice neurons by eA $\beta$ os depends on APP cleavage by $\gamma$-secretase and $\beta$-secretase. $\boldsymbol{A}$, Representative traces of NMDA sEPSCs at $\mathrm{T}_{0}$ and $\mathrm{T}_{20}$ with DAPT $(5 \mu \mathrm{m})$; with DAPT $(5 \mu \mathrm{m})$ plus eA $\beta$ os. $\boldsymbol{B}$, Bar graphs (mean \pm SEM) showing the $T_{20} / T_{0}$ ratio of NMDA sEPSCs amplitude and frequency with DAPT (white bars; $n=7$ neurons, $N=4$ mice, Wilcoxon $W=4(16,-12), p=0.8125$, for sEPSCs amplitude; and Wilcoxon $W=-4(12,-16), p=$ 0.8125 , for sEPSCs frequency); with DAPT plus eA $\beta$ os (gray bars; $n=10$ neurons, $N=5$ mice, Wilcoxon $W=1(11,-10)$, $p>0.9999$, for sEPSCs amplitude; and Wilcoxon $W=-43(6,-49), p=0.0273$, for sEPS(s frequency). DAPT versus DAPT plus eA $\beta$ os (Mann-Whitney $U=33(61,92), p=0.8868$, for sEPSCs amplitude; and Mann-Whitney $U=30.5(67.5,85.5), p=$ 0.6874 , for sEPSCs frequency). C, Representative traces of NMDA sEPSCs at $\mathrm{T}_{0}$ and $\mathrm{T}_{20}$ with $\beta$-secretase inhibitor $(1 \mu \mathrm{m})$; with $\beta$-secretase inhibitor $(1 \mu \mathrm{M})$ plus eA $\beta$ 0s. $D$, Bar graphs (mean \pm SEM) showing the $\mathrm{T}_{20} / \mathrm{T}_{0}$ ratio of NMDA sEPSC amplitude and frequency with $\beta$-secretase inhibitor (white bars; $n=6$ neurons, $N=4$ mice, Wilcoxon $\mathrm{W}=1(11,-10), p>0.9999$, for SEPSCS amplitude; and Wilcoxon $W=1(11,-10), p>0.9999$, for sEPSCs frequency); with $\beta$-secretase inhibitor plus eA $\beta$ os (gray bars; $n=8$ neurons, $N=3$ mice, Wilcoxon $W=12(24,-12), p=0.4609$, for sEPSCs amplitude; and Wilcoxon $W=$ $-36(0,-36), p=0.0078$, for sEPSCs frequency). $\beta$-Secretase inhibitor versus $\beta$-secretase inhibitor plus eA $\beta$ os (MannWhitney $U=17(38,67), p=0.4136$, for sEPSC amplitude; and Mann-Whitney $U=5(64,41), p=0.0127$, for sEPSC frequency). ${ }^{*} p<0.05 ; \# p<0.05$, \#\#p <0.01 relative to the $\mathrm{T}_{0}$ recording normalized to $100 \%$.

the presence of DAPT ( $5 \mu \mathrm{M}), \gamma$-secretase activity was reduced by $70 \%$ when compared with APP695-Gal4 alone $(p<0.0001$; $n=4$ independent experiments; Fig. $6 A$ ). These results validate the $\gamma$-secretase test to measure the effect of eA $\beta$ os on APP processing. When we applied eA $\beta$ os to N2a cells expressing APP695-Gal4, we observed a time-dependent increase in luciferase activity (T60 vs control: $148 \pm 2.47 \%$; $p<0.0001 ; n=4$ independent experiments; Fig. $6 B$ ) but not with eA $\beta$ monomers (T60 vs control: $105.46 \pm 1.04 \% ; p=0.26 ; n=3$ independent experiments; Fig. $6 B$ ). This result indicates that eA $\beta$ os enhance APP processing by $\gamma$-secretase. Different binding sites for A $\beta$ have been described on APP (Van Nostrand et al., 2002; Khalifa et al., 2010), and it has been shown that A $\beta$-APP interaction leads to APP dimerization (Fogel et al., 2014). This process promotes APP processing and $\mathrm{A} \beta$ production by the $\gamma$-secretase (Scheuermann et al., 2001; Munter et al., 2007). We tested whether sAPP interferes with the eA $\beta$ os-induced processing of APP. We preincubated N2a cells expressing APP695-Gal4 with increasing concentration of SAPP (0.1, 1 , and $5 \mathrm{~nm}$ ) and then applied eA $\beta$ os for $1 \mathrm{~h}$ (Fig. 6C). We observed a dosedependent inhibition in $\gamma$-secretasemediated APP processing in the presence of sAPP ( $\mathrm{T}_{60}$ vs sAPP 5 nM: $105.9 \pm 1.16 \% ; p<0.0001 ; n=3$ independent experiments). These data confirmed that eA $\beta$ os enhance the amyloidogenic processing of APP and suggest that sAPP may compete with $\mathrm{A} \beta$ for binding on APP, thus limiting $\mathrm{A} \beta$-induced APP processing as suggested by a previous publication (Gralle et al., 2009). However, we cannot exclude that sAPP sequesters eA $\beta$ os and subsequently reduced the effect of eA $\beta$ os rather than exerting a direct competition between eA $\beta$ os and sAPP for binding to APP.

\section{Alteration of NMDA sEPSC amplitudes by eAßos depends on APP cleavage by $\gamma$-secretase and \\ $\beta$-secretase}

We then tested in cortical slice neurons whether perturbations of NMDAdependent synaptic transmission by eA $\beta$ os require APP processing. We first inhibited APP cleavage by inhibiting $\gamma$-secretase with DAPT added in the $\mathrm{Mg}^{2+}$-free ACSF. When we perfused this inhibitor alone at a concentration of $5 \mu \mathrm{M}$, we did not detect any modifications of either NMDA sEPSC amplitudes $\left(\mathrm{T}_{20}\right.$ vs $\mathrm{T}_{0}: 102.67 \pm 3.51 \% ; p=$ $0.81 ; n=7$ neurons; $N=4$ mice) or frequencies $\left(\mathrm{T}_{20}\right.$ vs $\mathrm{T}_{0}: 91.29 \pm 22.97 \%$; $p=0.81 ; n=7$ neurons; $N=4$ mice; Fig. $7 A, B)$. In contrast, the reduction of NMDA sEPSC amplitudes induced by $\mathrm{eA} \beta$ os was abolished in the presence of DAPT $\left(\mathrm{T}_{0}=38.89 \pm 7.3 \mathrm{pA} ; \mathrm{T}_{20}=\right.$ $44.53 \pm 13.75 \mathrm{pA} ; \mathrm{T}_{20}$ vs $\mathrm{T}_{0}: 113.07 \pm$ $16.12 \% ; p>0.9999 ; n=10$ neurons; $N=5$ mice), while we still observed a reduction of NMDA sEPSC frequencies $\left(\mathrm{T}_{0}=\right.$ $0.61 \pm 0.14 \mathrm{~Hz} ; \mathrm{T}_{20}=0.42 \pm 0.10 \mathrm{~Hz} ; \mathrm{T}_{20}$ vs $\mathrm{T}_{0}: 73.08 \pm 9.61 \%$; $p=0.027 ; n=10$ neurons; $N=5$ mice; Fig. $7 A, B)$.

Because $\gamma$-secretase is involved in both amyloidogenic and nonamyloidogenic pathways, we investigated the pivotal role of APP processing in the effect of eA $\beta$ os by performing experiments with the $\beta$-secretase inhibitor added in the $\mathrm{Mg}^{2+}$-free ACSF. At a concentration of $1 \mu \mathrm{M}, \beta$-secretase inhibitor affected neither NMDA sEPSC amplitudes $\left(\mathrm{T}_{20}\right.$ vs $\mathrm{T}_{0}$ : $98.85 \pm 20.68 \%$; $p>0.9999 ; n=6$ neurons; $N=3$ mice) nor frequencies $\left(\mathrm{T}_{20} \mathrm{vs}\right.$ 
$\mathrm{T}_{0}: 102.58 \pm 12.17 \% ; p>0.9999 ; n=6$ neurons; $N=4$ mice; Fig. $7 C, D)$. The reduction of NMDA sEPSC amplitudes induced by eA $\beta$ os was abolished when we inhibited $\beta$-secretase cleavage of APP $\left(\mathrm{T}_{0}=32.91 \pm 6.15 \mathrm{pA} ; \mathrm{T}_{20}=35.11 \pm 5.71 \mathrm{pA} ; \mathrm{T}_{20}\right.$ vs $\mathrm{T}_{0}$ : $112.67 \pm 11.62 \% ; p=0.46 ; n=8$ neurons; $N=3$ mice), while we still observed a reduction of NMDA sEPSC frequencies $\left(\mathrm{T}_{0}=\right.$ $0.74 \pm 0.09 \mathrm{~Hz} ; \mathrm{T}_{20}=0.39 \pm 0.09 \mathrm{~Hz} ; \mathrm{T}_{20}$ vs $\mathrm{T}_{0}: 52.14 \pm 8.39 \%$; $p=0.007 ; n=8$ neurons; $N=3$ mice; Fig. $7 C, D)$. Thus, the alteration of NMDA sEPSC amplitudes by eA $\beta$ os involved APP processing toward the amyloidogenic pathway.

\section{eAßos inhibition of long-term potentiation requires}

\section{$\boldsymbol{\beta}$-secretase cleavage of APP}

Activity-dependent facilitation of excitatory synaptic transmission is a prevalent mechanism of synaptic plasticity underlying learning and memory processes in the mammalian brain. This is particularly well documented in the CA1 region of the hippocampus (Malenka and Nicoll, 1999). For instance, theta burst stimulation protocol produces an NMDA-dependent LTP at CA3-CA1 synapses (Grover et al., 2009). Since several studies reported that hippocampal LTP is highly sensitive to $A \beta$ os, we investigated whether this synaptic plasticity perturbation induced by $\mathrm{eA} \beta$ os requires APP processing toward the amyloidogenic pathway. We applied a theta burst stimulation protocol to induce LTP at CA3-CA1 synapses on acute hippocampal slices from WT mice. eA $\beta$ os applied 20 min before theta burst stimulation significantly reduced the potentiation (for the last $5 \mathrm{~min}$ of recording: $130.09 \pm 5.78$ in eA $\beta$ os condition; $n=12$ slices; $N=8$ mice; gray circle) compared with the control condition $(200.83 \pm 15.14 \% ; p=0.0003 ; n=11$ slices; $N=6$ mice; Fig. $8 A$, white circle). When we applied the $\beta$-secretase inhibitor IV ( 1 $\mu \mathrm{M})$, we prevented the alteration of LTP induced by eA $\beta$ os (183.63 $\pm 11.35 ; p=0.021 ; n=8$ slices; $N=4$ mice; gray triangle), while this inhibitor had no effect by itself when perfused $20 \mathrm{~min}$ before theta burst stimulation (194.38 $\pm 13.48 ; p=0.96$; $n=9$ slices; $N=5$ mice; Fig. $8 B$, white triangle). These results demonstrated that LTP perturbation induced by eA $\beta$ os in the CA1 region of the hippocampus involved $\beta$-secretase cleavage of APP.

\section{eAßos-induced processing of APP leads to accumulation of} cytosolic Aßos

Several studies reported that extracellular A $\beta$ os application promotes a production followed by an accumulation of $\mathrm{A} \beta$ inside the neurons (Yang et al., 1999; Tampellini et al., 2009), and that intraneuronal accumulation of $\mathrm{A} \beta$ is toxic and precedes its extracellular deposition in patients and mouse models of $\mathrm{AD}$ (Gouras et al., 2000; Wirths et al., 2001; Takahashi et al., 2002; Oddo et al., 2006). Accordingly, we tested whether eA $\beta$ os could modify the processing of APP and lead to cytosolic accumulation of APP fragments (Fig. 9). To visualize the processing of APP, we transfected primary cortical neurons with fluorescently tagged APP construct (mcherry-APPswe-EYFP). In this construction, mCherry fluorescent protein was inserted into the ectodomain and YFP fused to the C-terminal domain of APP695 expressing the Swedish mutation (APPswe). Thus, unprocessed APP or partially processed APP ( $\beta$-secretase cleavage only) appeared in yellow while the presence of red or green puncta reveals processed APP. The location of APP processing to produce A $\beta$ peptides has been ascribed to many organelles, including the endoplasmic recticulum, the trans-Golgi network, endosomes, and lysosomes (Small and Gandy, 2006; Thinakaran and Koo, 2008). These organelles serve as sorting stations for
A

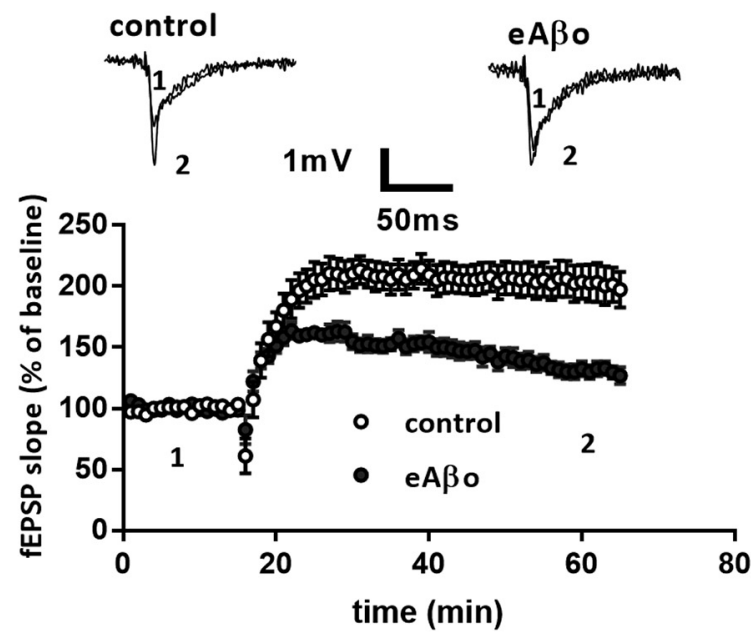

B
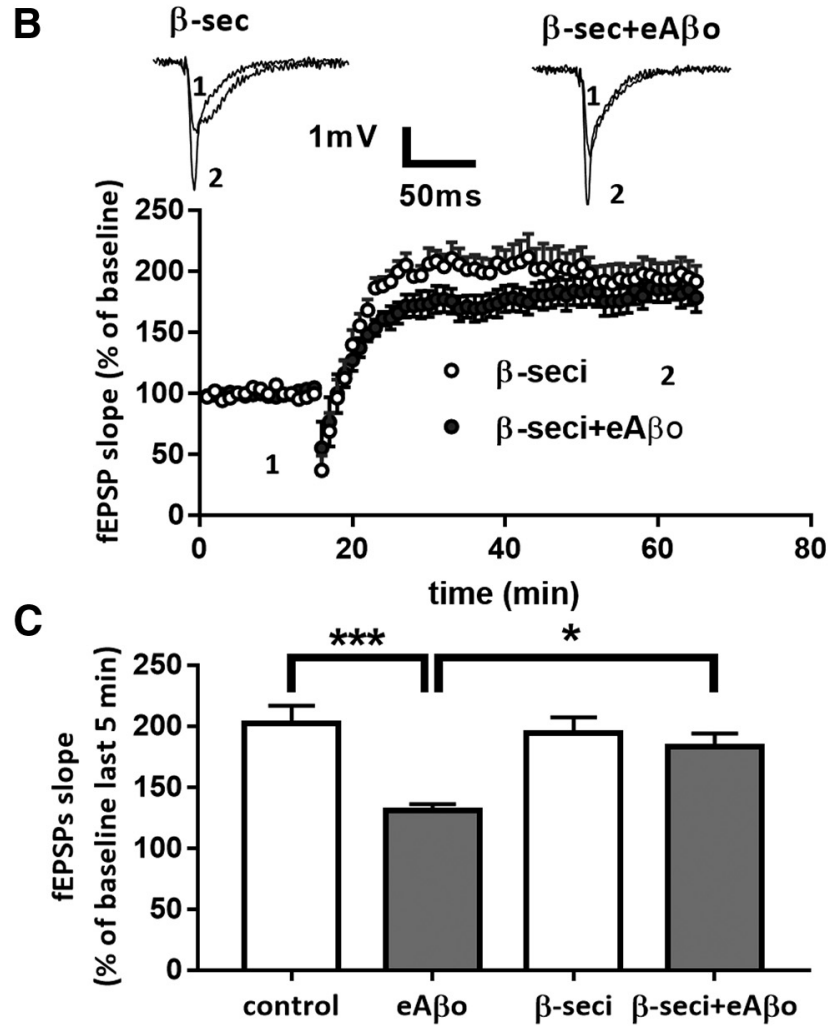

Figure 8. $\quad \beta$-Secretase inhibition prevents the long-term plasticity inhibition induced by $\mathrm{eA} \beta$ os. $\boldsymbol{A}$, Data are the mean ( \pm SEM), and they are expressed as percentages of fEPSP slope baseline in the control condition (white circle; $n=11$ slices, $N=6$ mice); with eA $\beta$ os (gray circle; $n=12$ slices, $N=8$ mice). Representative traces from one experiment are shown. They were extracted at the times indicated $(1,2)$ on the graph. $\boldsymbol{B}$, Data are the mean ( \pm SEM), and they are expressed as percentages of fEPSP slope baseline with $\beta$-secretase inhibitor ( $1 \mu \mathrm{m}$; white triangle; $n=9$ slices, $N=5$ mice); with $\beta$-secretase inhibitor plus eA $\beta$ os ( $1 \mu \mathrm{m}$; gray triangle; $n=8$ slices, $N=4$ mice). Representative traces from one experiment are shown. They were extracted at the times indicated $(1,2)$ on the graph. $\boldsymbol{C}$, Summary bar graph depicting the effect of various experimental conditions on LTP. On the graph, data are the mean ( \pm SEM), and they are expressed as percentages of fEPSP slope baseline measured during the 5 last min of recordings in control condition (white bar); with eA $\beta$ os (gray bar); with $\beta$-secretase inhibitor (white bar); and with $\beta$-secretase inhibitor plus eA $\beta$ os (gray bar). One-way ANOVA and Tukey's post hoc test for multiple comparisons: $F_{(3,36)}=8.288, p=0.0003$; control vs eA $\beta$ os, $p=0.0003$; eA $\beta$ os vs $\beta$-seci $+\mathrm{eA} \beta$ os, $p=0.0218$. ${ }^{*} p<0.05$, ${ }^{* * *} p<0.001$ 
A

control
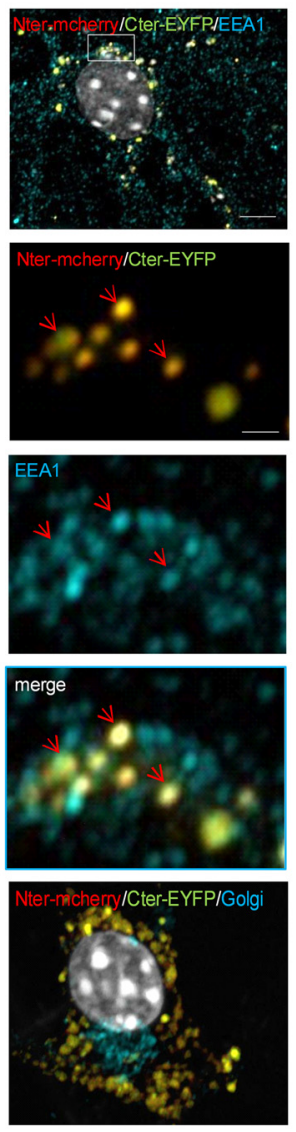

eA $\beta$ o
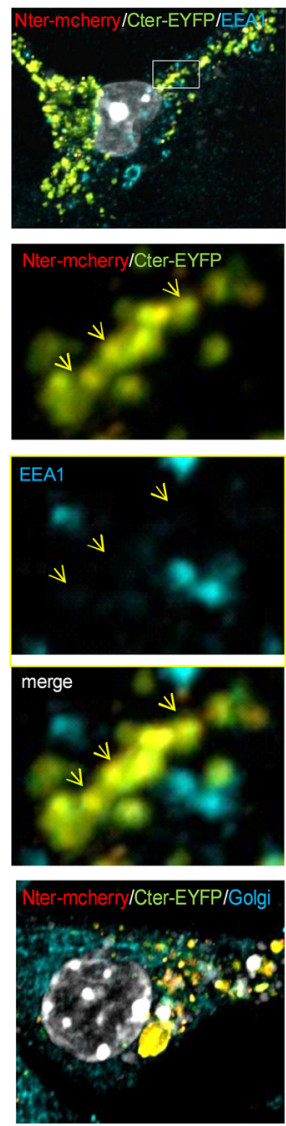

control
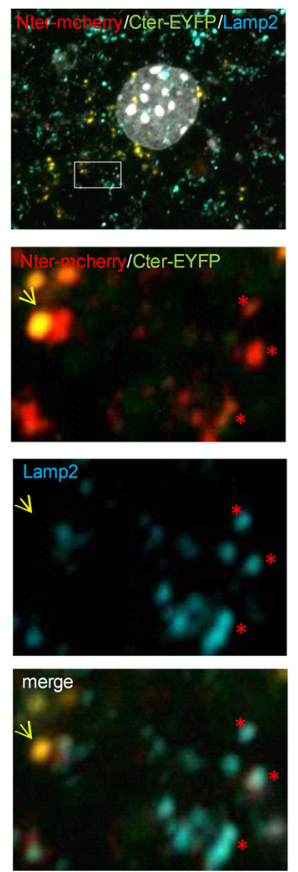

B
eA $\beta$ o
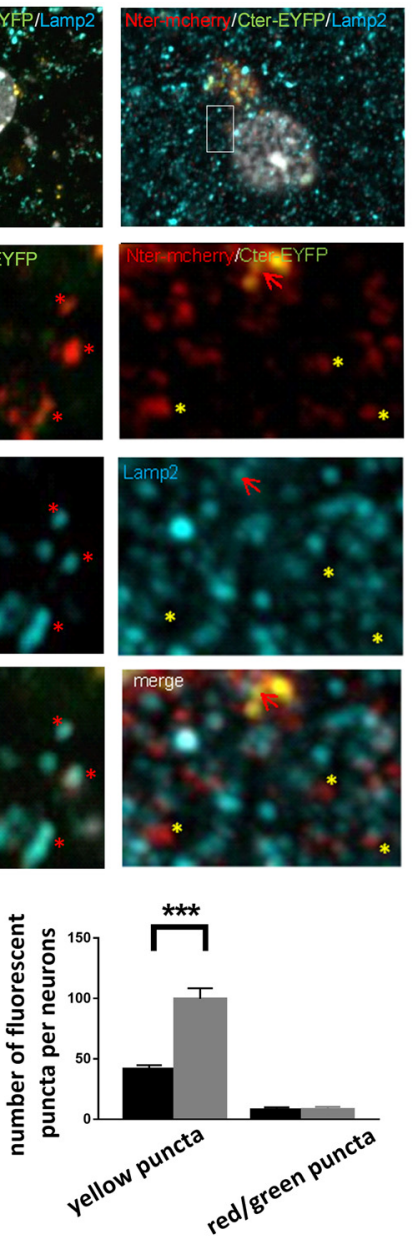

D

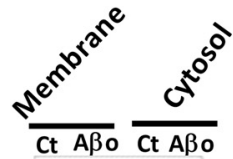
(22C11) APP (110 kDa)
SAPP (95 kDa)

Ct $A B O$

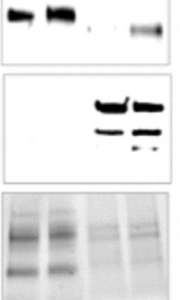

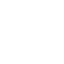

(4G8)

Loading

Figure 9. eA $\beta$ os-induced processing of APP leads to the accumulation of cytosolic A $\beta$ oligomers. $\boldsymbol{A}$, Airyscan images of cortical neurons transfected with mcherry-APPsw-EYFP, treated or not with eA $\beta$ os for $30 \mathrm{~min}$ and immunostained with EEA1, LAMP2, and 58K Golgi protein antibodies (blue).White rectangle areas indicate higher magnification used for fluorescent puncta quantification. Red arrows and red stars indicate respectively colocalized yellow puncta or red puncta with EEA1 or LAMP2 vesicles, yellow arrows, and yellow stars indicate, respectively, noncolocalized yellow puncta or red puncta with EEA1 or LAMP2 vesicles. Wide-field scale bar, $5 \mu \mathrm{m}$. Magnification scale bar, $1 \mu \mathrm{m}$. $\boldsymbol{B}$, Number of yellow puncta or red/green puncta in cortical neurons treated (gray bars) or not (black bars) with eA $\beta$ os. For yellow puncta, one-way ANOVA and Tukey's post hoc test for multiple comparisons $\left(F_{(3,44)}=83.58, p<0.0001\right.$; control vs eA $\beta 0$, $p<0.001$. C, Proportion of yellow or red/green puncta in the different subcellular compartments in cortical neurons treated (gray bars) or not (black bars) with eA $\beta$ os. For yellow puncta, one-way ANOVA and Tukey's post hoc test for multiple comparisons $\left(F_{(5,35)}=7.35, p<0.0001\right.$; for early endosomes: control vs eA $\beta 0$ s, $p=0.0481$; for Golgi apparatus: control vs eA $\beta$ os, $p=0.0495)$. For red/green puncta, one-way ANOVA and Tukey's post hoc test for multiple comparisons $\left(F_{(5,29)}=7.089, p=0.0002\right.$; for Golgi apparatus: control vs eA $\beta 05, p=0.0017$. ${ }^{*} p<0.05,{ }^{* *} p<0.01,{ }^{* * *} p<0.001$. Data are presented as the mean \pm SEM. $\boldsymbol{D}$, Western blot analysis of APP full-length and APP fragments in membrane or cytosol fractions obtained from cortical neurons exposed or not with eA $\beta$ os for $30 \mathrm{~min}$.

proteins that traffic through the endocytic and secretory pathways. To characterize the site of APP processing, we performed confocal analysis of the fluorescence obtained with specific markers for early endosomes (EEA1), lysosomes (Lamp2), and the Golgi apparatus (58K Golgi protein; Fig. 9B). In presence of
$\mathrm{eA} \beta$ os, the number of yellow puncta per neuron significantly increased compared with control $(99.7 \pm 8.70$ vs $41.6 \pm 3.14$; $p<0.001 ; n=10-14$ ), while there was no significant difference regarding the red/green puncta (Fig. $9 C$ ). In control cells, yellow puncta localized predominantly to the early endosome as 
visualized by the EEA1 labeling (39.80 \pm $11.11 \%$ ) with only a subset of yellow puncta colocalized with the lysosome marker Lamp2 $(4.15 \pm 1.48 \%)$ or the Golgi marker $58 \mathrm{~K}(7.065 \pm 2.37 \%$; Fig. $9 A, C)$. In eA $\beta$ ostreated neurons, yellow puncta were redistributed from early endosomes to Golgi apparatus (for early endosomes: $39.80 \pm$ $11.11 \%$ in control cells vs $13.72 \pm 6.58 \%$ in eA $\beta$ os-treated cells, $p=0.0481$; for Golgi: $7.065 \pm 2.37 \%$ in control cells vs $34.50 \pm$ $6.35 \%$ in eA $\beta$ os-treated cells, $p=0.0495$ $n=5-10$ ). Concerning processed APP (red/ green puncta), we observed that only $2 \%$ are colocalized with early endosomes. The processing of APP occurred preferentially in lysosomes in control conditions (20.57 \pm $8.08 \%$ vs $6.93 \pm 2.49 \%$ in eA $\beta$ os-treated cells) and in Golgi apparatus in the presence of eA $\beta$ os $(62.92 \pm 20.24 \%$ vs $0 \%$ in control cells; $p=0.0017 ; n=5-9)$. These data indicated that eA $\beta$ os lead to APP accumulation and processing in the Golgi apparatus.

Next, we investigated the consequences of APP processing in the production of soluble/cytosolic A $\beta$ peptides. Control or treated cells were permeabilized with digitonin. The two resulting protein fractions (membrane-bound and soluble/cytosolic) were collected separately and analyzed by Western blot, using 22C11 (directed against the $\mathrm{N}$ terminal of APP) and 4G8 (directed against $\mathrm{A} \beta$ ) antibodies (Fig. 9D).While APP was found in membrane-bound fractions, sAPP appeared in the soluble/cytosolic fractions of eA $\beta$ os-treated neurons as revealed by the $22 \mathrm{C} 11$ antibodies. Furthermore, we also observed an accumulation of $\mathrm{A} \beta$ oligomers (trimers and tetramers forms) and a band at $50 \mathrm{kDa}$ that may represent large SDS-stable oligomers in the soluble/cytosol fractions of eA $\beta$ os-treated neurons compared with control. Together, these data provide evidence that $\mathrm{eA} \beta$ os led to APP processing through the secretory pathway and to a cytosolic accumulation of $\mathrm{A} \beta$ oligomers.

Application of intracellular A $\beta$ oligomers perturbs spontaneous synaptic activity in cultures of mouse cortical neurons

From these observations, we decided to evaluate the effects of cytosolic accumulation of $\mathrm{A} \beta$ os on both AMPA/kainate and NMDA-dependent synaptic transmission by infusing $A \beta$ os directly into the cytosol of neurons via the patch pipette. We infused $A \beta$ os at $300 \mathrm{~nm}$ into cultured neurons through the recording patch pipette (iA $\beta$ os). In this condition, we recorded sEPSCs in $\mathrm{Mg}^{2+}$-free ACSF at $\mathrm{T}_{0}$ and $\mathrm{T}_{20}$. After $20 \mathrm{~min}$ of iA $\beta$ os, we did not observe any modifications of AMPA/kainate sEPSC amplitudes and frequencies $\left(\mathrm{T}_{0}=21.65 \pm 1.89 \mathrm{pA} ; \mathrm{T}_{20}=\right.$ $23.50 \pm 4.65 \mathrm{pA} ; \mathrm{T}_{20}$ vs $\mathrm{T}_{0}: 103.93 \pm 13.1 \% ; p=0.96 ; n=11$ neurons; and $\mathrm{T}_{0}=0.69 \pm 0.11 \mathrm{~Hz} ; \mathrm{T}_{20}=0.65 \pm 0.14 \mathrm{~Hz} ; \mathrm{T}_{20}$ vs $\mathrm{T}_{0}$ : $108.93 \pm 27.6 \% ; p=0.46 ; n=11$ neurons, respectively; Fig. $10 \mathrm{~A}$,
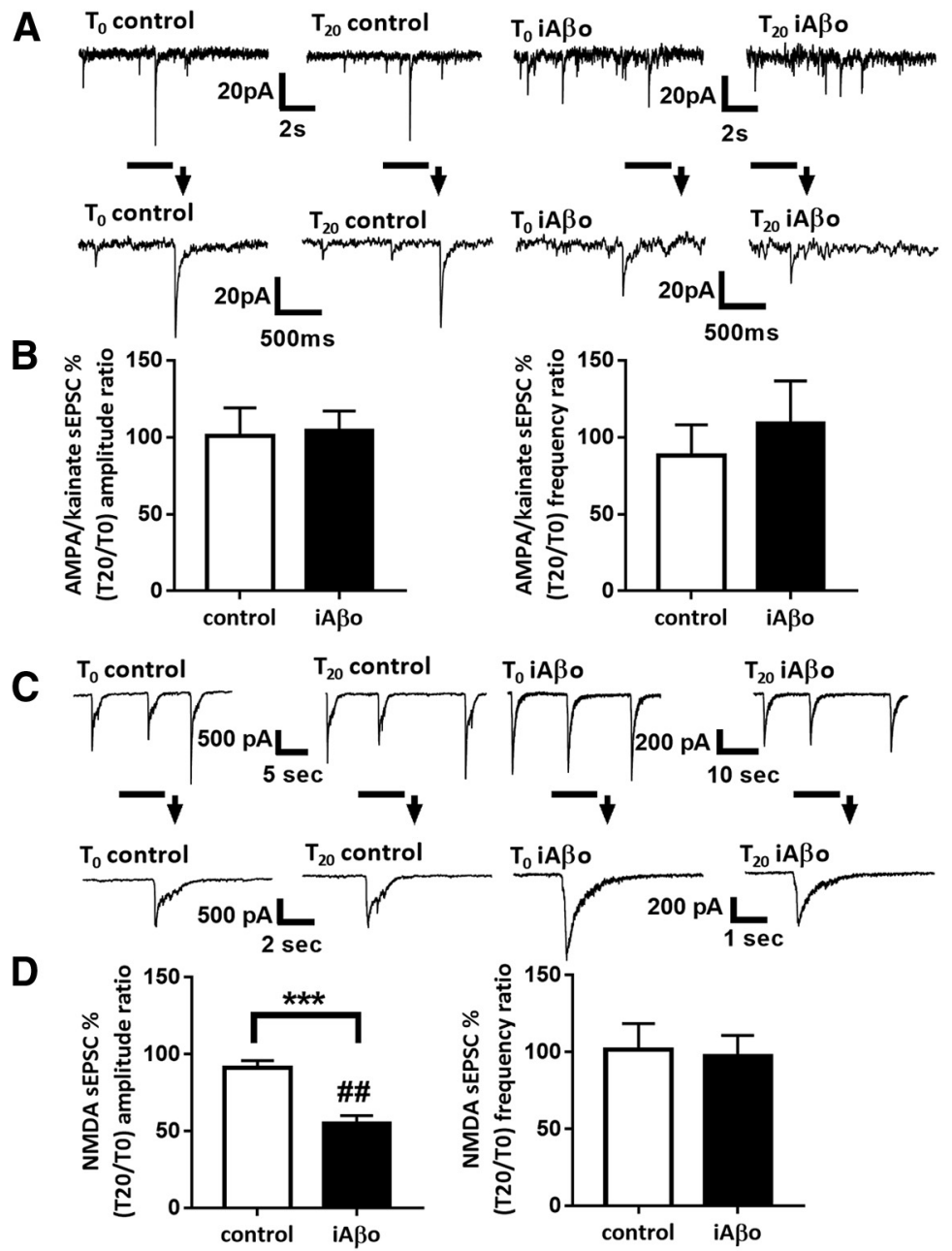

Figure 10. iA $\beta$ os perturb spontaneous synaptic activity in cultures of mouse cortical neurons. $\boldsymbol{A}$, Representative traces of AMPA/NMDA sEPSCs at $\mathrm{T}_{0}$ and $\mathrm{T}_{20}$ in control condition or with $\mathrm{iA} \beta 0 \mathrm{~S}$ ( $\left.300 \mathrm{nM}\right)$. $\boldsymbol{B}$, Bar graphs (mean \pm SEM) se $\mathrm{T}_{20} / \mathrm{T}_{0}$ ratio of AMPA/kainate $S E P S C$ amplitude and frequency in control condition (white bars; $n=10$ neu(Mann-Whitney $U=47(102,129), p=0.6047$, for sEPSC amplitudes; and Mann-Whitney $U=50(105,126)$, $p=0.7561$, for sEPSC frequencies). $C$, Representative traces of NMDA sEPSCS at $T_{0}$ and $T_{20}$ in control condition; with iA $\beta$ os (300 nm). D, Bar graphs (mean \pm SEM) showing the $T_{20} / T_{0}$ ratio of NMDA sEPSC amplitudes and frequencies in control condition (white bars; $n=13$ neurons, Wilcoxon $W=-55(18,-73), p=0.0574$, for sEPSC amplitudes; and Wicoxon $W=-21(12,-33), p=0.2383$, for sEPSC frequencies); with iA $\beta$ os (black bars, $n=9$ neurons, Wilcoxon quencies). Control versus iA $\beta$ os for NMDA sEPSCs (Mann-Whitney $U=6(202,51), p=0.0001$, for sEPSC amplitudes; and Mann-Whitney $U=54.5(145.5,107.5), p=0.8041$, for sEPSC frequencies). ${ }^{* * *} p<0.001$; \#\#p $<0.01$, relative to the $\mathrm{T}_{0}$ recording normalized to $100 \%$.

$B)$. In contrast, $\mathrm{iA} \beta$ os reduced NMDA sEPSC amplitudes $\left(\mathrm{T}_{0}=\right.$ $681.06 \pm 72.23 \mathrm{pA} ; \mathrm{T}_{20}=375.06 \pm 54.32 \mathrm{pA} ; \mathrm{T}_{20}$ vs $\mathrm{T}_{0}: 54.50 \pm$ $5.1 \% ; p=0.0039 ; n=9$ neurons) without affecting NMDA sEPSC frequencies $\left(\mathrm{T}_{0}=0.10 \pm 0.02 \mathrm{~Hz} ; \mathrm{T}_{20}=0.10 \pm 0.02 \mathrm{~Hz} ; \mathrm{T}_{20}\right.$ vs $\mathrm{T}_{0}$ : $97.36 \pm 13.5 \% ; p=0.71 ; n=9$ neurons; Fig. $10 C, D)$. Similar to eA $\beta$ os, 20 min of iA $\beta$ os induced a strong reduction of NMDA but not of AMPA/kainate sEPSC amplitudes in primary cultures of cortical neurons.

iA $\beta$ os reduce NMDA current amplitudes in cortical slice neurons from both WT and APP KO mice

Next, we checked whether iA $\beta$ os-induced alterations of excitatory neurotransmission in cortical slice neurons from WT and 

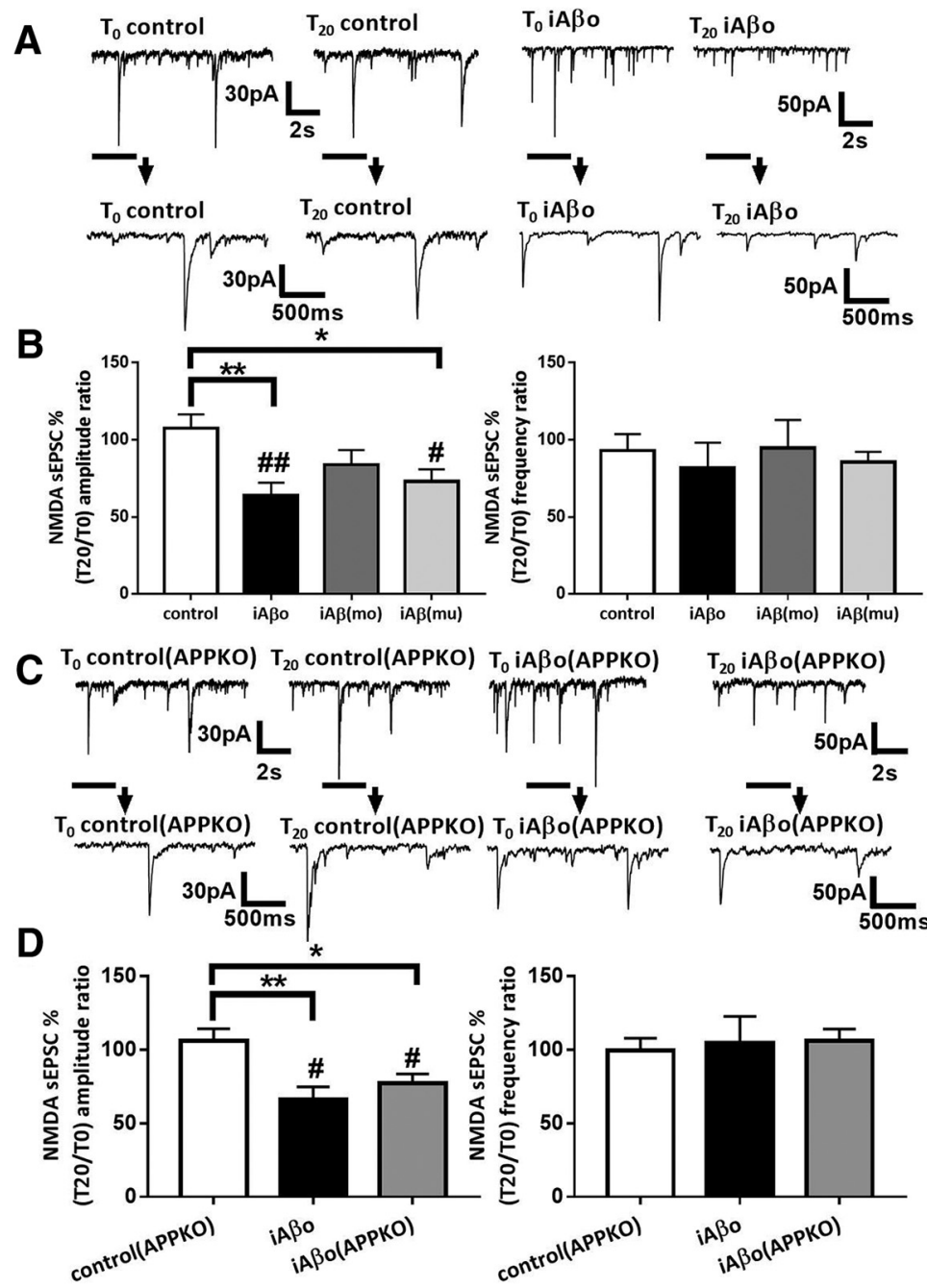

Figure 11. iA $\beta$ Os reduces NMDA currents amplitude in cortical slice neurons from both WT and APP KO mice. $\boldsymbol{A}_{\text {, }}$ Representative traces of NMDA sEPSCs recorded in WT neurons from Swiss mice at $T_{0}$ and $T_{20}$ in control condition; with iA $\beta$ os. $B$, Bar graphs (mean \pm SEM) showing the $T_{20} / T_{0}$ ratio of NMDA sEPSC amplitude and frequency recorded in WT neurons from Swiss mice in control condition (white bars; $n=11$ neurons, $N=9$ mice, Wilcoxon $W=22(44,-22)$, $p=0.3652$, for sEPSC amplitudes; and Wilcoxon $W=-16(25,-41), p=0.5195$, for $s E P S C$ frequencies); with iA $\beta 0$ s (black bars; $n=8, N=6$ mice, Wilcoxon $W=-36(0,-36), p=0.0078$, for sEPSC amplitudes; and Wilcoxon $W=-14$ $(11,-25), p=0.3828$, for sEPSC frequencies); with iA $\beta$ mo (gray bars; $n=8, N=4$ mice, Wilcoxon $W=-31(12,-43)$, $p=0.1309$, for sEPSC amplitudes; and Wilcoxon $W=-9(23,-32), p=0.6953$, for sEPSC frequencies); and with murine $\mathrm{iA} \beta$ (iA $\beta$ (mu)), $300 \mathrm{~nm}$; gray bar; $n=9$ neurons, $N=4$ mice, Wilcoxon $\mathrm{W}=-39(42,-3), p=0.0195$, for sEPSC amplitudes; and Wilcoxon $W=26(31,-5), p=0.0781$, for sEPSC frequencies). One-way ANOVA and Tukey's post hoc test for multiple comparisons $\left(F_{(3,34)}=4.555, p=0.0087\right.$; control vs iA $\beta 0$ s, $p=0.0090$; control vs (iA $\beta(\mathrm{mu})$ ), $p=0.0412$, for NMDA sEPSCS amplitudes) and one-way ANOVA followed by Tukey's post hoc test for multiple comparisons $\left(F_{(3,34)}=\right.$ $0.1971, p=0.8977$ for NMDA sEPSC frequencies). C, Representative traces of NMDA sEPSCs recorded in APP K0 neurons at $\mathrm{T}_{0}$ and $\mathrm{T}_{20}$ in control condition, with $\mathrm{iA} \beta$ OS. $\boldsymbol{D}$, Bar graphs (mean \pm SEM) showing the $\mathrm{T}_{20} / \mathrm{T}_{0}$ ratio of NMDA sEPSC amplitudes and frequencies recorded in APP KO neurons in control condition (white bars; $n=6$ neurons, $N=6$ mice, Wilcoxon $W=1(11,-10), p>0.9999$, for sEPSC amplitudes; and Wilcoxon $W=-3(9,-12), p=0.8438$, for sEPSC frequencies);

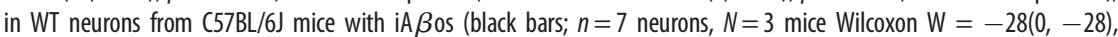
$p=0.0156$, for sEPSC amplitudes; and Wilcoxon W $=3(12,-9), p=0.8438$, for sEPSC frequencies), and APP KO neurons with iA $\beta$ os (gray bars; $n=8$ neurons, $N=3$ mice, Wilcoxon $\mathrm{W}=-32(2,-34), p=0.0234$, for $\mathrm{SEPSC}$ amplitudes; and Wilcoxon $W=10(23,-13), p=0.5469$, for sEPSCS frequencies). One-way ANOVA and Tukey's post hoc test for multiple comparisons $\left[F_{(2,18)}=6.754, p=0.0065\right.$; control(APP K0) vs iA $\beta 0$ s, $p=0.0058$; control(APP K0) vs iA $\beta$ os (APP K0), $p=0,0382$, for NMDA sEPSC amplitudes; and one-way ANOVA followed by Tukey's post hoc test for multiple comparisons $\left(F_{(2,18)}=0.07,348, p=0.9294\right.$, for NMDA sEPSC frequencies). ${ }^{*} p<0.05$, ${ }^{* *} p<0.01$; \#p $<0.05$, \#\#p $<0.01$ relative to the $\mathrm{T}_{0}$ recording normalized to $100 \%$.

APP KO mice. iA $\beta$ os reduced NMDA sEPSC amplitudes $\left(\mathrm{T}_{0}=\right.$ $36.30 \pm 6.27 \mathrm{pA} ; \mathrm{T}_{20}=22.29 \pm 5.43 \mathrm{pA} ; \mathrm{T}_{20}$ vs $\mathrm{T}_{0}: 63.80 \pm 8.2 \%$; $p=0.007 ; n=8$ neurons; $N=6$ mice) without affecting NMDA sEPSC frequencies $\left(\mathrm{T}_{0}=0.68 \pm 0.14 \mathrm{~Hz} ; \mathrm{T}_{20}=0.61 \pm 0.22 \mathrm{~Hz}\right.$;
$\mathrm{T}_{20}$ vs $\mathrm{T}_{0}: 81.67 \pm 16.4 \% ; p=0.38 ; n=8$ neurons; $N=6$ mice; Fig. $11 A, B$ ) recorded in cortical slice neurons from Swiss WT mice. In a dose-response experiment, we observed that the reduction induced by iA $\beta$ os was still observed at $50 \mathrm{~nm}$ but not at $10 \mathrm{~nm}$ (data not shown). This reduction of NMDA sEPSC amplitudes seemed to require $\mathrm{A} \beta$ os since we failed to observe any modifications of NMDA currents when neurons were infused with a solution containing $\mathrm{A} \beta$ monomers (300 nм; $\mathrm{T}_{0}=$ $35.05 \pm 7.00 \mathrm{pA} ; \mathrm{T}_{20}=24.32 \pm 3.58 \mathrm{pA} ; \mathrm{T}_{20}$ vs $\mathrm{T}_{0}: 83.75 \pm 9.6 \% ; p=0.13 ; n=10$ neurons; $N=4$ mice; Fig. $11 B$ ). These findings strengthened our previous observation that eA $\beta$ os effects are due to iA $\beta$ os accumulation originating from the processing of murine APP. Subsequently, because the murine $\mathrm{A} \beta$ peptide exhibits a different sequence than human $\mathrm{A} \beta$, we tested the infusion of murine $\mathrm{A} \beta$ oligomers ( $300 \mathrm{~nm}$ ) on NMDA-dependent sEPSCs. Similar to human $\mathrm{A} \beta$ os, we observed a reduction of the amplitudes $\left(\mathrm{T}_{0}=32.33 \pm 3.21 \mathrm{pA}\right.$; $\mathrm{T}_{20}=22.14 \pm 1.74 \mathrm{pA} ; \mathrm{T}_{20}$ vs $\mathrm{T}_{0}: 72.86 \pm$ $8.02 \% ; p=0.019 ; n=9$ neurons; $N=4$ mice) but not of the frequencies $\left(\mathrm{T}_{0}=\right.$ $0.51 \pm 0.06 \mathrm{~Hz} ; \mathrm{T}_{20}=0.43 \pm 0.06 \mathrm{~Hz} ; \mathrm{T}_{20} \mathrm{vs}$ $\mathrm{T}_{0}: 85.60 \pm 6.6 \% ; 0 . p=0.078 ; n=9$ neurons; $N=4$ mice; Fig. $11 B$ ) of NMDA-dependent sEPSCs recorded in neurons infused with murine $\mathrm{A} \beta$ os (iA $\beta(\mathrm{mu}))$. In an additional set of experiments, we tested the influence of intracellular accumulation of $\mathrm{A} \beta$ os in APP KO mice. Interestingly, $\mathrm{iA} \beta$ os also affected the amplitudes $\left(\mathrm{T}_{0}=35.49 \pm\right.$ $5.07 \mathrm{pA} ; \mathrm{T}_{20}=24.10 \pm 4.03 \mathrm{pA} ; \mathrm{T}_{20}$ vs $\mathrm{T}_{0}$ : $77,10 \pm 6.39 \% ; p=0.023 ; n=8$ neurons; $N=3$ mice), but not the frequencies $\left(\mathrm{T}_{0}=\right.$ $0.63 \pm 0.12 \mathrm{~Hz} ; \mathrm{T}_{20}=0.64 \pm 0.10 \mathrm{~Hz} ; \mathrm{T}_{20}$ vs $\mathrm{T}_{0}: 105.96 \pm 8.03 \% ; p=0.54 ; n=8$ neurons; $N=3$ mice; Fig. $11 C, D)$ of sEPSCs recorded in cortical slice neurons from APP KO mice. These data demonstrated that a cytosolic accumulation of $\mathrm{A} \beta$ os significantly affected NMDA-dependent synaptic transmission in both neuronal cultures and cortex slices.

Intracellular infusion of cortical neurons with an antibody directed against

$A \beta$ prevents the inhibition of

NMDA-dependent synaptic

transmission induced by eAßos

Since murine iA $\beta$ os promoted a decrease of NMDA sEPSC amplitudes, we investigated whether eA $\beta$ os-induced reduction of NMDA synaptic transmission was linked to an accumulation of newly produced endogenous $\mathrm{A} \beta \mathrm{os}$. For this purpose, the 4G8 antibody directed against the sequence 17-24 of $\mathrm{A} \beta$ peptide was infused in the neurons through the recording patch pipette. The 4G8 antibody 
has been shown previously to completely prevent the block of LTP by $\mathrm{A} \beta$ os through a rapid and direct neutralization of the peptide (Klyubin et al., 2005). Remarkably, 4G8 antibody (1:100, $10 \mu \mathrm{g} /$ $\mathrm{ml}$ ) infusion into cortical slice neurons affected neither NMDA sEPSC amplitudes $\left(\mathrm{T}_{20}\right.$ vs $\mathrm{T}_{0}: 91.22 \pm 4.5 \% ; p=0.68 ; n=6$ neurons; $N=3$ mice) nor frequencies $\left(\mathrm{T}_{20}\right.$ vs $\mathrm{T}_{0}$ : $91.31 \pm 3 \% ; p=0.43 ; n=6$ neurons; $N=3$ mice), whereas it prevented the inhibition of NMDA sEPSC amplitudes induced by eA $\beta$ os $\left(\mathrm{T}_{0}=30.36 \pm 6.74 \mathrm{pA}\right.$; $\mathrm{T}_{20}=29.09 \pm 5.96 \mathrm{pA} ; \mathrm{T}_{20}$ vs $\mathrm{T}_{0}: 97.13 \pm$ $2.52 \% ; p=0.195 ; n=8$ neurons; $N=6$ mice), but not the frequencies reduction $\left(\mathrm{T}_{0}=0.90 \pm 0.24 \mathrm{~Hz} ; \quad \mathrm{T}_{20}=0.50 \pm\right.$ $0.16 \mathrm{~Hz} ; \mathrm{T}_{20}$ vs $\mathrm{T}_{0}: 58.49 \pm 3.71 \% ; p=$ $0.023 ; n=8$ neurons; $N=6$ mice; Fig. $12 A$, $B)$. These data suggested that NMDA current alterations induced by eA $\beta$ os depend on newly produced $\mathrm{A} \beta$ that accumulates intracellularly after APP processing.

\section{Discussion}

The impact of extracellular $\mathrm{A} \beta$ os on excitatory synaptic transmission has been extensively studied to further understand the mechanism of action of $\mathrm{A} \beta \mathrm{os}$ in the disruption of learning and memory processes associated with $\mathrm{AD}$. If a consensus has emerged that validates the idea that $\mathrm{A} \beta$ os application negatively affects excitatory neurotransmission, questions remain about the molecular mechanism involved.

In our study, we demonstrated that $20 \mathrm{~min}$ of eA $\beta$ os significantly affect glutamatergic synaptic transmission by inducing a selective decrease of NMDA sEPSC amplitudes and frequencies. The reduction of NMDA sEPSC amplitudes but not the frequencies requires the presence of APP and the activity of $\beta$ - and $\gamma$-secretases, two enzymes involved in the amyloidogenic pathway. The processing of APP driven by eA $\beta$ os promotes a subsequent accumulation of $A \beta$ os into the cytosol, leading to the reduction of synaptic NMDA currents.

Among the potential membrane receptors of $A \beta$ os (JaroszGriffiths et al., 2016), several reports described that the peptide interacts with the extracellular domain of APP (Shaked et al., 2006, 2009; Fogel et al., 2014; Puzzo et al., 2017). Such an interaction may occur in our model since eA $\beta$ os-induced $\gamma$-secretase activity is abolished by the extracellular application of $\operatorname{sAPP} \alpha$ that can interact with $\mathrm{A} \beta$ os and compete with its binding on APP. These results are strengthened by the observation that the effects of eA $\beta$ os on NMDA sEPSCs are prevented in cortical neurons from APP KO mice. In a recent article, Wang et al. (2017) demonstrate that $\mathrm{A} \beta$ os-containing $\mathrm{AD}$ brain extracts induced blockade of hippocampal LTP and depends on APP expression. Several studies have described a selective reduction of NMDA receptor function by $\mathrm{A} \beta$ os via a decreased membrane expression of memory-related NMDA receptors (Snyder et al., 2005), even if a direct reduction of NMDA receptor function (Chen and Roche, 2007) without alterations of GluN2A and GluN2B expression at the synaptic level cannot be excluded (Frandemiche et al., 2014). Our results also showed that mutant APP overexpression, which leads to increased production of $\mathrm{A} \beta$ in the extracellular space induces synaptotoxicity in the nearby "healthy" neurons. This effect seemed to be proportional to the amount of $\mathrm{A} \beta$ produced. Indeed, APPs we-overexpressing neurons, which display an increased production of $\mathrm{A} \beta$ when compared with APPwt-overexpressing neurons, induced a more pronounced synaptotoxic effect on the healthy neuron. These results suggest that a relationship exists between the neuronal production of $\mathrm{A} \beta$ and the severity of the synaptotoxic effect observed in healthy neurons. When we conducted the same experiments using APP KO cortical neurons, the healthy APP KO neuron was not affected by the nearby A $\beta$-secreting neuron. This suggests that the effects observed on the healthy wild-type neuron require APP expression. Together, these data reveal that genetic ablation of APP prevents A $\beta$ os-mediated synaptic dysfunction. Subsequently, we showed that the effect of eA $\beta$ os on NMDA currents requires the presence of not only APP but also its cleavage through the amyloidogenic pathway. Indeed, the impacts of eA $\beta$ os on NMDA sEPSC amplitudes and LTP in the hippocampus are reversed by treatment with $\gamma$ - and/or $\beta$-secretase inhibitors, highlighting a causal role of the amyloidogenic pathway in these processes and strengthening the therapeutic interest of the pharmacological blockade of the APP processing pathway. Our observations are strengthened by a biochemical assay in which we observed that eA $\beta$ os promote APP processing toward the amyloidogenic pathway, revealing a vicious circle triggered by eA $\beta$ os that may contribute to the transmission of the disease from sick to healthy neurons. In a recent article, Puzzo et al. (2017), also confirm the requirement of APP expression in A $\beta$ os-mediated synaptic dysfunction even if APP processing does not seem to be involved. In the amyloidogenic pathway, APPs are internalized into endocytic compartments and subsequently cleaved to generate $\mathrm{A} \beta$. In pathologic conditions, the accumulation of $\mathrm{A} \beta$ os inside the endocytic system perturbs the function and induces a loss of membrane permeability of endosomal/lysosomal compartments (Yang et al., 1998; Willén et al., 2017). The loss of membrane permeability is correlated 
with a release of $\mathrm{A} \beta$ os into the cytosol (Yang et al., 1998). Our data highlight another mechanism in which eA $\beta$ os also promote APP processing during the secretory pathway resulting in the accumulation of cytosolic $\mathrm{A} \beta$ oligomers (Fig. 7). From these observations, we evaluated the effects of cytosolic $A \beta$ os accumulation by infusing $\mathrm{A} \beta$ os (300 nM) directly into the cytosol of neurons via the patch pipette. We observed that, similar to eA $\beta$ os, $20 \mathrm{~min}$ of iA $\beta$ os induce a reduction of NMDA sEPSC amplitudes in neurons from both cultures and acute cortical slices. This effect was still detected at $50 \mathrm{~nm}$ iA $\beta$ os but lost at $10 \mathrm{~nm}$ (data not shown). These results demonstrate that a cytosolic infusion of $\mathrm{A} \beta$ os may disrupt synaptic glutamatergic transmission and subsequently the ability of neurons to induce synaptic plasticity in $\mathrm{AD}$ pathology. The implication of intracellular $\mathrm{A} \beta$ os in the physiopathological pathways that sustain $\mathrm{AD}$-related cellular alteration has been identified in many animal models of AD. Consistent with our data, previous reports related that $\mathrm{A} \beta$ os application on neuronal culture promotes production and intracellular accumulation of A $\beta$ (Yang et al., 1999; Tampellini et al., 2009). Similarly, the Osaka (E613Del) mutation of APP identified in a Japanese pedigree showing Alzheimer's-type dementia is associated with massive intracellular $\mathrm{A} \beta$ os accumulation that impairs organelle transport and induces dramatic dendritic spine loss in neurons likely contributing to synaptic pathology in $\mathrm{AD}$ (Umeda et al., 2015). How intracellular accumulation of $A \beta$ os perturb these neuronal functions remains to be fully demonstrated. Several studies described the presence of intracellular $\mathrm{A} \beta$ and recently, high-resolution imaging techniques have localized $\mathrm{A} \beta$ oligomers inside postsynaptic densities of excitatory synapses in $\mathrm{AD}$ mouse models (Gouras et al., 2010; Capetillo-Zarate et al., 2011; Pickett et al., 2016) that may uncouple NMDA receptors function and/or synaptic expression.

The findings showing that reduction of current frequencies was not normalized by treatment with inhibitors of $\mathrm{A} \beta$-producing secretases suggest that eA $\beta$ os may exert differential effects on the presynaptic and postsynaptic compartments of the synapse. Indeed, our data reveal that eA $\beta$ os reduced the frequency of spontaneous but not miniature AMPA/kainate EPSCs recorded in the presence of TTX, suggesting an alteration of neuronal excitability. Perturbations of cellular excitability have already been reported in transgenic models of $\mathrm{AD}$ or after $\mathrm{A} \beta \mathrm{os}$ application. Indeed, it has been shown that $\mathrm{A} \beta$-overproducing transgenic mice present a reduction in somatic $\mathrm{Na}^{+}$current (Brown et al., 2011) or an increase in $\mathrm{K}^{+}$maximal conductance (Tamagnini et al., 2015). Similarly, in another transgenic mouse model of $\mathrm{AD}$, neurons involved in the major input to the entorhinal cortex exhibited a decreased firing frequency during depolarization, as well as an increased spike frequency adaptation (Marcantoni et al., 2014). Thus, a perturbation of action potential properties might lead to the $\mathrm{A} \beta$ os-driven reduction of sEPSC frequencies described in our study, but additional experiments will be required to confirm this hypothesis. More particularly, it was reported that $\mathrm{A} \beta$ oligomers modulate voltage-gated calcium and potassium channels directly or indirectly, by changing the properties of the membrane (Lioudyno et al., 2012). Thus, a direct effect of eA $\beta$ os on sodium or potassium channels could reduce sEPSC frequencies through a perturbation of action potential properties.

In summary, our study suggests that APP processing promoted by eA $\beta$ os is followed by cytosolic accumulation of $\mathrm{A} \beta$ oligomers responsible for the alteration of glutamatergic neurotransmission. These results strengthen the point of view that synaptic dysfunction in $\mathrm{AD}$ is closely linked to the accumulation of intracellular $\mathrm{A} \beta$ os. The result showing that the intracellular infusion of 4G8 antibody inhibits the negative impact of eA $\beta$ os reinforces this scheme. Moreover, APP processing also produces a releasable pool of $A \beta$ os into the extracellular space that may contribute to the transfer of pathology from pathologic to healthy neurons and to a larger scale to the spreading of $\mathrm{AD}$ in different brain structures. Finally, it will be necessary to further determine how the $\mathrm{iA} \beta$-dependent reduction of NMDAR sEPSC amplitude is responsible for $\mathrm{A} \beta$-induced cognitive dysfunction in Alzheimer's disease. Pharmacological strategies targeting this functional relationship between extracellular and intracellular $\mathrm{A} \beta$ os may represent a novel therapeutic approach to the early stage of the disease.

\section{References}

Bayer TA, Wirths O (2010) Intracellular accumulation of amyloid-beta-a predictor for synaptic dysfunction and neuron loss in Alzheimer's disease. Front Aging Neurosci 2:8.

Brown JT, Chin J, Leiser SC, Pangalos MN, Randall AD (2011) Altered intrinsic neuronal excitability and reduced $\mathrm{Na}+$ currents in a mouse model of Alzheimer's disease. Neurobiol Aging 32:2109.e1-14.

Capetillo-Zarate E, Gracia L, Yu F, Banfelder JR, Lin MT, Tampellini D, Gouras GK (2011) High-resolution 3D reconstruction reveals intra-synaptic amyloid fibrils. Am J Pathol 179:2551-2558.

Chen BS, Roche KW (2007) Regulation of NMDA Receptors by Phosphorylation. Neuropharmacology 53:362-368.

Cipriani G, Dolciotti C, Picchi L, Bonuccelli U (2011) Alzheimer and his disease: a brief history. Neurol Sci 32:275-279.

Florean C, Zampese E, Zanese M, Brunello L, Ichas F, De Giorgi F, Pizzo P (2008) High content analysis of $\gamma$-secretase activity reveals variable dominance of presenilin mutations linked to familial Alzheimer's disease. Biochim Biophys Acta 1783:1551-1560.

Fogel H, Frere S, Segev O, Bharill S, Shapira I, Gazit N, O’Malley T, Slomowitz E, Berdichevsky Y, Walsh DM, Isacoff EY, Hirsch JA, Slutsky I (2014) APP homodimers transduce an amyloid- $\beta$-mediated increase in release probability at excitatory synapses. Cell Rep 7:1560-1576.

Frandemiche ML, De Seranno S, Rush T, Borel E, Elie A, Arnal I, Lanté F, Buisson A (2014) Activity-dependent tau protein translocation to excitatory synapse is disrupted by exposure to amyloid-beta oligomers. J Neurosci 34:6084-6097.

Goldstein JM, Litwin LC (1993) NBQX is a selective non-NMDA receptor antagonist in rat hippocampal slice. Mol Chem Neuropathol 18:145-152.

Gouras GK, Tsai J, Naslund J, Vincent B, Edgar M, Checler F, Greenfield JP, Haroutunian V, Buxbaum JD, Xu H, Greengard P, Relkin NR (2000) Intraneuronal $A \beta 42$ accumulation in human brain. Am J Pathol 156:1520.

Gouras GK, Tampellini D, Takahashi RH, Capetillo-Zarate E (2010) Intraneuronal beta-amyloid accumulation and synapse pathology in Alzheimer's disease. Acta Neuropathol 119:523-541.

Gralle M, Botelho MG, Wouters FS (2009) Neuroprotective secreted amyloid precursor protein acts by disrupting amyloid precursor protein dimers. J Biol Chem 284:15016-15025.

Greenfield JP, Tsai J, Gouras GK, Hai B, Thinakaran G, Checler F, Sisodia SS, Greengard P, Xu H (1999) Endoplasmic reticulum and trans-Golgi network generate distinct populations of Alzheimer beta-amyloid peptides. Proc Natl Acad Sci U S A 96:742-747.

Grover LM, Kim E, Cooke JD, Holmes WR (2009) LTP in hippocampal area CA1 is induced by burst stimulation over a broad frequency range centered around delta. Learn Mem 16:69-81.

Haass C, Kaether C, Thinakaran G, Sisodia S (2012) Trafficking and proteolytic processing of APP. Cold Spring Harb Perspect Med 2:a006270.

Hoey SE, Williams RJ, Perkinton MS (2009) Synaptic NMDA receptor activation stimulates $\alpha$-secretase amyloid precursor protein processing and inhibits amyloid- $\beta$ production. J Neurosci 29:4442-4460.

Jarosz-Griffiths HH, Noble E, Rushworth JV, Hooper NM (2016) Amyloid$\beta$ receptors: the good, the bad, and the prion protein. J Biol Chem 291:3174-3183. 
Kamenetz F, Tomita T, Hsieh H, Seabrook G, Borchelt D, Iwatsubo T, Sisodia S, Malinow R, Point W (2003) APP processing and synaptic function. Neuron 37:925-937.

Khalifa NB, Van Hees J, Tasiaux B, Huysseune S, Smith SO, Constantinescu SN, Octave JN, Kienlen- Campard P (2010) What is the role of amyloid precursor protein dimerization? Cell Adh Migr 4:268-272.

Klyubin I, Walsh D, Lemere CA, Cullen WK, Shankar GM, Betts V, Spooner ET, Jiang L, Anwyl R, Selkoe DJ, Rowan MJ (2005) Amyloid $\beta$ protein immunotherapy neutralizes $\mathrm{A} \beta$ oligomers that disrupt synaptic plasticity in vivo. Nat Med 11:556-561.

LaFerla FM, Green KN, Oddo S (2007) Intracellular amyloid- $\beta$ in Alzheimer's disease. Nat Rev Neurosci 8:499-509.

Léveillé F, El Gaamouch F, Gouix E, Lecocq M, Lobner D, Nicole O, Buisson A (2008) Neuronal viability is controlled by a functional relation between synaptic and extrasynaptic NMDA receptors. FASEB J 22:4258-4271.

Li S, Hong S, Shepardson NE, Walsh DM, Shankar GM, Selkoe D (2009) Soluble oligomers of amyloid Beta protein facilitate hippocampal longterm depression by disrupting neuronal glutamate uptake. Neuron 62:788-801.

Lioudyno MI, Broccio M, Sokolov Y, Rasool S, Wu J, Alkire MT, Liu V, Kozak JA, Dennison PR, Glabe CG, Lösche M, Hall JE (2012) Effect of synthetic $\mathrm{A} \beta$ peptide oligomers and fluorinated solvents on Kv1.3 channel properties and membrane conductance. PLoS One 7:e35090.

Lorenzo A, Yuan M, Zhang Z, Paganetti PA, Sturchler-Pierrat C, Staufenbiel M, Mautino J, Vigo FS, Sommer B, Yankner BA (2000) Amyloid beta interacts with the amyloid precursor protein: a potential toxic mechanism in Alzheimer's disease. Nat Neurosci 3:460-464.

Malenka RC, Nicoll RA (1999) Long-term potentiation a decade of progress? Science 285:1870-1874.

Marcantoni A, Raymond EF, Carbone E, Marie H (2014) Firing properties of entorhinal cortex neurons and early alterations in an Alzheimer's disease transgenic model. Pflugers Arch 466:1437-1450.

Munter LM, Voigt P, Harmeier A, Kaden D, Gottschalk KE, Weise C, Pipkorn R, Schaefer M, Langosch D, Multhaup G (2007) GxxxG motifs within the amyloid precursor protein transmembrane sequence are critical for the etiology of $A \beta 42$. EMBO J 26:1702-1712.

Näslund J, Schierhorn A, Hellman U, Lannfelt L, Roses AD, Tjernberg LO, Silberring J, Gandy SE, Winblad B, Greengard P (1994) Relative abundance of Alzheimer $\mathrm{A} \beta$ amyloid peptide variants in Alzheimer disease and normal aging. Proc Natl Acad Sci U S A 91:8378-8382.

Oddo S, Caccamo A, Shepherd JD, Murphy MP, Golde TE, Kayed R, Metherate R, Mattson MP, Akbari Y, LaFerla FM (2003) Triple-transgenic model of Alzheimer's disease with plaques and tangles: intracellular Abeta and synaptic dysfunction. Neuron 39:409-421.

Oddo S, Caccamo A, Smith IF, Green KN, LaFerla FM (2006) A dynamic relationship between intracellular and extracellular pools of Abeta. Am J Pathol 168:184-194.

Pickett EK, Koffie RM, Wegmann S, Henstridge CM, Herrmann AG, Colom-Cadena M, Lleo A, Kay KR, Vaught M, Soberman R, Walsh DM, Hyman BT, Spires-Jones TL (2016) Non-fibrillar oligomeric amyloid- $\beta$ within synapses. J Alzheimers Dis 53:787-800.

Puzzo D, Piacentini R, Fá M, Gulisano W, Li Puma DD, Staniszewski A, Zhang H, Tropea MR, Cocco S, Palmeri A, Fraser P, D’Adamio L, Grassi C, Arancio O (2017) LTP and memory impairment caused by extracellular A $\beta$ and Tau oligomers is APP-dependent. Elife 6:e26991.

Sannerud R, Declerck I, Peric A, Raemaekers T, Menendez G, Zhou L, Veerle B, Coen K, Munck S, De Strooper B, Schiavo G Annaert W (2011) ADP ribosylation factor 6 (ARF6) controls amyloid precursor protein (APP) processing by mediating the endosomal sorting of BACE1. Proc Natl Acad Sci U S A 108:E559-E568.

Scheuermann S, Hambsch B, Hesse L, Stumm J, Schmidt C, Beher D, Bayer TA, Beyreuther K, Multhaup G (2001) Homodimerization of amyloid precursor protein and its implication in the amyloidogenic pathway of Alzheimer's disease. J Biol Chem 276:33923-33929.

Shaked GM, Kummer MP, Lu DC, Galvan V, Bredesen DE, Koo EH (2006) Abeta induces cell death by direct interaction with its cognate extracellular domain on APP (APP 597-624). FASEB J 20:1254-1256.
Shaked GM, Chauv S, Ubhi K, Hansen LA, Masliah E (2009) Interactions between the amyloid precursor protein C-terminal domain and G proteins mediate calcium dysregulation and amyloid beta toxicity in Alzheimer's disease. FEBS J 276:2736-2751.

Shankar GM, Bloodgood BL, Townsend M, Walsh DM, Selkoe DJ, Sabatini B (2007) Natural oligomers of the Alzheimer amyloid- $\beta$ protein induce reversible synapse loss by modulating an NMDA-type glutamate receptordependent signaling pathway. J Neurosci 27:2866-2875.

Small SA, Gandy S (2006) Sorting through the cell biology of Alzheimer's disease: intracellular pathways to pathogenesis. Neuron 52:15-31.

Snyder EM, Nong Y, Almeida CG, Paul S, Moran T, Choi EY, Nairn AC, Salter MW, Lombroso PJ, Gouras GK, Greengard P (2005) Regulation of NMDA receptor trafficking by amyloid-beta. Nat Neurosci 8:1051-1058.

Stine WB Jr, Dahlgren KN, Krafft GA, LaDu MJ (2003) In vitro characterization of conditions for amyloid-beta peptide oligomerization and fibrillogenesis. J Biol Chem 278:11612-11622.

Takahashi RH, Milner TA, Li F, Nam EE, Edgar MA, Yamaguchi H, Beal MF, Xu H, Greengard P, Gouras GK (2002) Intraneuronal Alzheimer abeta42 accumulates in multivesicular bodies and is associated with synaptic pathology. Am J Pathol 161:1869-1879.

Tamagnini F, Novelia J, Kerrigan TL, Brown JT, Tsaneva-Atanasova K, Randall AD (2015) Altered intrinsic excitability of hippocampal CA1 pyramidal neurons in aged PDAPP mice. Front Cell Neurosci 9:372.

Tampellini D, Rahman N, Gallo EF, Huang Z, Dumont M, Capetillo-Zarate E, Ma T, Zheng R, Lu B, Nanus DM, Lin MT, Gouras GK (2009) Synaptic activity reduces intraneuronal Abeta, promotes APP transport to synapses, and protects against $\mathrm{A} \beta$-related synaptic alterations. J Neurosci 29:9704-9713

Thinakaran G, Koo EH (2008) Amyloid precursor protein trafficking, processing, and function. J Biol Chem 283:29615-29619.

Traynelis SF, Wollmuth LP, McBain CJ, Menniti FS, Vance KM, Ogden KK, Hansen KB, Hongjie Y, Myers SJ, Dingledine R (2010) Glutamate receptor ion channels: structure, regulation, and function. Pharmacol Rev 62:405-496.

Umeda T, Ramser EM, Yamashita M, Nakajima K, Mori H, Silverman MA, Tomiyama T (2015) Intracellular amyloid $\beta$ oligomers impair organelle transport and induce dendritic spine loss in primary neurons. Acta Neuropathol Commun 3:51.

Van Nostrand WE, Melchor JP, Keane DM, Saporito-Irwin SM, Romanov G, Davis J, Xu F (2002) Localization of a fibrillar amyloid beta-protein binding domain on its precursor. J Biol Chem 277:36392-36398.

Walsh DM, Klyubin I, Fadeeva JV, Cullen WK, Anwyl R, Wolfe MS, Rowan MJ, Selkoe DJ (2002) Naturally secreted oligomers of amyloid beta protein potently inhibit hippocampal long-term potentiation in vivo. Nature 416:535-539.

Wang Z, Jackson RJ, Hong W, Taylor WM, Corbett GT, Moreno A, Liu W, Li S, Frosch MP, Slutsky I, Young-Pearse TL, Spires-Jones TL, Walsh DM (2017) Human brain-derived A $\beta$ oligomers bind to synapses and disrupt synaptic activity in a manner that requires APP. J Neurosci 37:11947-11966.

Wei W, Nguyen LN, Kessels HW, Hagiwara H, Sisodia S, Malinow R (2010) Amyloid beta from axons and dendrites reduces local spine number and plasticity. Nat Neurosci 13:190-196.

Willén K, Edgar JR, Hasegawa T, Tanaka N, Futter CE, Gouras GK (2017) A $\beta$ accumulation causes MVB enlargement and is modelled by dominant negative VPS4A. Mol Neurodegener 12:61.

Wirths O, Multhaup G, Czech C, Blanchard V, Moussaoui S, Tremp G, Pradier L, Beyreuther K, Bayer TA (2001) Intraneuronal Abeta accumulation precedes plaque formation in beta-amyloid precursor protein and presenilin-1 double-transgenic mice. Neurosci Lett 306:116-120.

Yang AJ, Chandswangbhuvana D, Margol L, Glabe CG (1998) Loss of endosomal/lysosomal membrane impermeability is an early event in amyloid A $\beta$ 1-42 pathogenesis. J Neurosci Res 52:691-698.

Yang AJ, Chandswangbhuvana D, Shu T, Henschen A, Glabe CG (1999) Intracellular accumulation of insoluble, newly synthesized abetan- 42 in amyloid precursor protein-transfected cells that have been treated with Abeta1-42. J Biol Chem 274:20650-20656. 